\title{
Enhancing Security in Multicellular Multicast Channels Reducing Interference Power with the Best Relay Selection
}

\author{
Shaikh Muhammad Rizwan Ali ${ }^{1}$, Md. Zahurul Islam Sarkar² \\ ${ }^{1}$ Department of Information and Communication Technology, BUP, Dhaka, Bangladesh \\ ${ }^{2}$ Department of Electrical and Electronic Engineering, RUET, Rajshahi, Bangladesh \\ Email: rizwan3358@student.bup.edu.bd, msarkar01@qub.ac.uk
}

How to cite this paper: Ali, S.M.R. and Sarkar, Md.Z.I. (2022) Enhancing Security in Multicellular Multicast Channels Reducing Interference Power with the Best Relay Selection. Journal of Computer and Communications, 10, 1-26. https://doi.org/10.4236/jcc.2022.101001

Received: December 9, 2021

Accepted: January 10, 2022

Published: January 13, 2022

Copyright $\odot 2022$ by author(s) and Scientific Research Publishing Inc. This work is licensed under the Creative Commons Attribution International License (CC BY 4.0).

http://creativecommons.org/licenses/by/4.0/

\section{(c) (i) Open Access}

\begin{abstract}
The capacity of wireless networks is fundamentally limited by interference. A few research has focused on the study of the simultaneous effect of interference and correlation, and less attention has been paid to the topic of canceling simultaneous effect of interference and correlation until recently. This paper considers a secure wireless multicasting scenario through multicellular networks over spatially correlated Nakagami- $m$ fading channel in the presence of multiple eavesdroppers. Authors are interested to protect the desired signals from eavesdropping considering the impact of perfect channel estimation (PCE) with interference and correlation. The protection of eavesdropping is also made strong reducing the simultaneous impact of interference and correlation on the secrecy multicast capacity employing opportunistic relaying technique. In terms of the signal-to-interference plus noise ratio (SINR), fading parameter, correlation coefficient, the number of multicast users and eavesdroppers and the number of antennas at the multicast users and eavesdroppers, the closed-form analytical expressions are derived for the probability of non-zero secrecy multicast capacity and the secure outage probability for multicasting to understand the insight of the effects of aforementioned parameters. The results show that the simultaneous effects of correlation and interference at the multicast users degrade security in multicasting. Moreover, the security in multicasting degrades with the intensity of fading and the number of multicast users, eavesdroppers and antennas at the eavesdroppers. The effects of these parameters on the security in multicasting can be significantly reduced by using opportunistic relaying technique with PCE. Finally, the analytical results are verified via Monte-Carlo simulation to justify the validity of derived closed-form analytical expressions.
\end{abstract}




\section{Keywords}

Correlation, Interference Power, Multicellular Multicast Channel, Nakagami- $m$ Fading, Opportunistic Relaying, Probability of Non-Zero

Secrecy Multicast Capacity, Secure Outage Probability for Multicasting

\section{Introduction}

An important limitation to the capacity of wireless communication systems is interference, which depends upon a number of factors including the locations of interfering transmitters. Although, the issue of interference has been studied extensively in the literature; however, much less attention has been paid to the topic of canceling simultaneous effect of the interference and correlation. The simultaneous effect of interference and correlation generally captures the fact that the interference created by interfering transmitters is a correlated stochastic process both spatially and temporally. It is well recognized that correlated fading reduces the performance gain in multi-antenna communications [1]. Likewise, it has recently been also proved that the simultaneous effect of interference and correlation decreases the diversity gain [2]. The simultaneous effect of interference and correlation partially comes from correlated channel attenuation, like correlated fading and shadowing.

Because of the randomness of the mobile propagation channels and limited radio spectrum, co-channel interference (CCI) and inter-symbol interference (ISI) are major impediments to high-capacity transmission in power and bandwidth-limited wireless communication systems. In cellular networks, CCI occurs from neighboring cells using the same radio channels. As the frequency reuse factor decreases from seven to three, then to one, CCI is unavoidable due to the channel reuse in adjacent cells. Given perfect knowledge of the channel coefficients of all co-channel signals, linear filter type receivers suppress CCI by controlling the filter coefficients in the sense of maximizing the signalto-interference-plus-noise ratio (SINR). In typical wireless mobile communication systems, CCI and ISI often arise together. Hence, the receiver designs for mitigating these impairments in joint fashions are quite common. In multiple-input multiple-output (MIMO) systems, large spectral efficiency can be achieved if the spatially multiplexed data streams, which manifest themselves as CCI to each other, are properly separated. Accordingly, CCI mitigation techniques developed for single antenna systems have been applied in decoding of multi-channel data streams. For the receivers with multiple antennas, diversity combining techniques broaden the freedom in interference mitigation receiver designs.

However, the impairments from CCI and ISI are the major obstacles to reliable communication in long-range cellular networks and in short-range wireless local and personal area networks. At present, linear filtering, equalization, and 
diversity combining techniques have been traditional means to combat the impairments in separate or joint fashions. Also, the interference canceling techniques designed for decoding of multiple single-user signals have been applied in decoding of spatially multiplexed data streams in MIMO systems. The impairments from high-power CCI and ISI in time-varying channels still impose severe constraints in the design of practical interference resilient receivers.

On the other hand, multicasting is an efficient wireless communication technique for group-oriented and personal communication such as video-conferencing, e-learning etc. Due to the increase of application areas and the mobility of users with network components, the security is a crucial aspect in wireless multicasting systems because of the fact that the medium of wireless multicasting is susceptible to eavesdropping and fraud [3].

\subsection{Related Works}

Recently, Shrestha et al. [4] studied secure wireless multicasting scenario through quasi-static Rayleigh fading channel in the presence of multiple eavesdroppers where each eavesdropper is equipped with multiple antennas. They showed that the physical layer security can be achieved even in the presence of multiple multi-antenna eavesdroppers. But they did not consider the effect of correlation and interference on the security in multicasting. In [5] [6], Giti et al. respectively used selective precoding and phase alignment precoding to enhance the security in multicasting by elimination interference power. But they did not consider the effect of correlation and the method that can be used to reduce the simultaneous effect of correlation and interference. Sayed et al. [7] studied secure wireless multicasting scenario through multi-cellular multiple-input multiple-output (MIMO) networks with linear equalization and investigated the effect of interference on the distributed and co-located MIMO channels. But they did not consider the effect of correlation and the method that can be used to reduce the effect of correlation and interference. An iterative algorithm was used by Nguyen et al. [8] to enhance the security in cooperative cognitive radio multicast networks. Opportunistic relaying technique was also used by Nguyen et al. [9] to study the security in half-duplex cognitive radio network. But they did not consider the effects of interference and correlation on the security analysis of multicasting. Kundu et al. [10] studied secure wireless multicasting through Generalized- $K$ fading channels and derived the closed-form analytical expressions for the probability of non-zero secrecy multicast capacity and the secure outage probability for multicasting without considering the effects of interference and correlation. Borshon et al. [11] investigated the effects of Weibull fading parameter on the security in multicasting. In [12], A. S. M. Badrudduza and M. K. Kundu investigated the effects of $\kappa-\mu$ fading parameters on the security in multicasting scenario. Atallah and Kaddoum [13] used partial cooperation to enhance the security in wireless networks in the presence of passive eavesdroppers. Sultana et al. [14] enhanced the security in cognitive radio multicast network using inter- 
ference power. But they did not consider the simultaneous effects of interference and correlation on the security of multicasting and did not use any technique to eliminate these effects. In [15], the opportunistic relaying technique was used by Kibria et al. to enhance the security in multicasting without considering the effects of interference and correlation. Sun et al. [16] investigated the impact of antenna correlation on the security of single-input multiple-output (SIMO) channel in the presence of multiple eavesdroppers. But they used an unicasting scenario to investigate that effect and did not consider the effect of interference on the multicasting network. In [17], Badrudduza et al. used opportunistic relaying technique to reduce the effect of correlation on the security in multicasting over Nakagami- $m$ fading channels. They did not consider the effect of interference and the method that can be used to reduce that effect. Moreover, the mathematical modeling of a system considering the simultaneous effect of correlation and interference is different than that of a system considering the effect of correlation and interference separately. Shahriyer et al. [18] used the same opportunistic relaying technique in enhancing security in multicasting over generalized fading channels. They considered the effect of shadowing but did not consider the effects of correlation and interference.

\subsection{Contributions}

Based on the aforementioned scenario available in the literature and motivated by the importance of security in practical multicasting, in this paper, authors studied a multicasting scenario through multi-cellular network and developed the mathematical model to ensure its security considering the simultaneous effect of correlation and interference. The major contributions of this paper can be summarized as follows.

- Based on the PDFs of SINRs of the source-to-relay, relay-to-destination and relay-to-eavesdropper links, at first, we derive the expressions for the PDFs of SINRs of the best relay for the multicast and eavesdropper channels in terms of the simultaneous effects of correlation and interference for Cell-1 and Cell-2.

- Secondly, using these PDFs of SINRs, we derive the analytical expressions for the probability of non-zero secrecy multicast capacity and the secure outage probability for multicasting to analyze the secure outage performance of the proposed model.

- Thirdly, we investigate the effect of correlation in the presence of interference and the effect of interference in the presence of correlation, and show how these effects can be reduced using opportunistic relaying technique.

- Finally, the derived analytical expressions have been justified via Monte-Carlo simulations.

The remainder of this paper is organized as follows. Sections II and III describe the system model and problem formulation, respectively. The expressions for the probability of non-zero secrecy multicast capacity and the secure outage 
probability for multicasting are derived respectively in Section IV and V. Numerical results are presented in Section VI. Finally, Section VII draws the conclusions of this work.

\section{System Model}

A secure wireless multicasting scenario shown in Figure 1 is considered through multicellular network over spatially correlated Nakagami- $m$ fading in the presence of multiple eavesdroppers. Two adjacent cells named "Cell-1" and "Cell-2" are considered to realize the effect of interferences. In Cell-1, base station-1 (BS-1) transmits a common stream of information to $M$ destination users via a group of $K$ relays $R_{k} \quad(k=1,2, \cdots, K)$ in the presence of $N$ eavesdroppers. Similarly, in Cell-2, base station-2 (BS-2) transmits a common stream of information to $L$ destination users via a group of $W$ relays $R_{w}(w=1,2, \cdots, W)$ in the presence of $Q$ eavesdroppers. All the relays, BS-1 and BS-2 and are equipped with single antenna. Each destination user and eavesdropper of Cell-1 are equipped with $n_{\eta_{1}}$ and $n_{e_{1}}$ antennas, respectively. While each multicast user and eavesdropper of Cell-2 are equipped with $n_{r_{2}}$ and $n_{e_{2}}$ antennas, respectively. The propagation channels are considered correlated and to be affected by the interference and Nakagami- $m$ fading. The correlation coefficients between two antennas of destination users and between two antennas of eavesdroppers of Cell-1 are denoted by $\rho_{m_{1}}$ and $\rho_{e_{1}}$, respectively. Similarly, the correlation coefficients between two antennas of destination users and between two antennas of eavesdroppers of Cell-2 are denoted by $\rho_{m_{2}}$ and $\rho_{e_{2}}$, respectively. Interferences at the destination users and eavesdroppers of Cell-1 are denoted by $I_{m_{1}}$ and $I_{e_{1}}$, respectively. Similarly, interferences at the destination users and eavesdroppers of Cell-2 are denoted by $I_{m_{2}}$ and $I_{e_{2}}$, respectively.

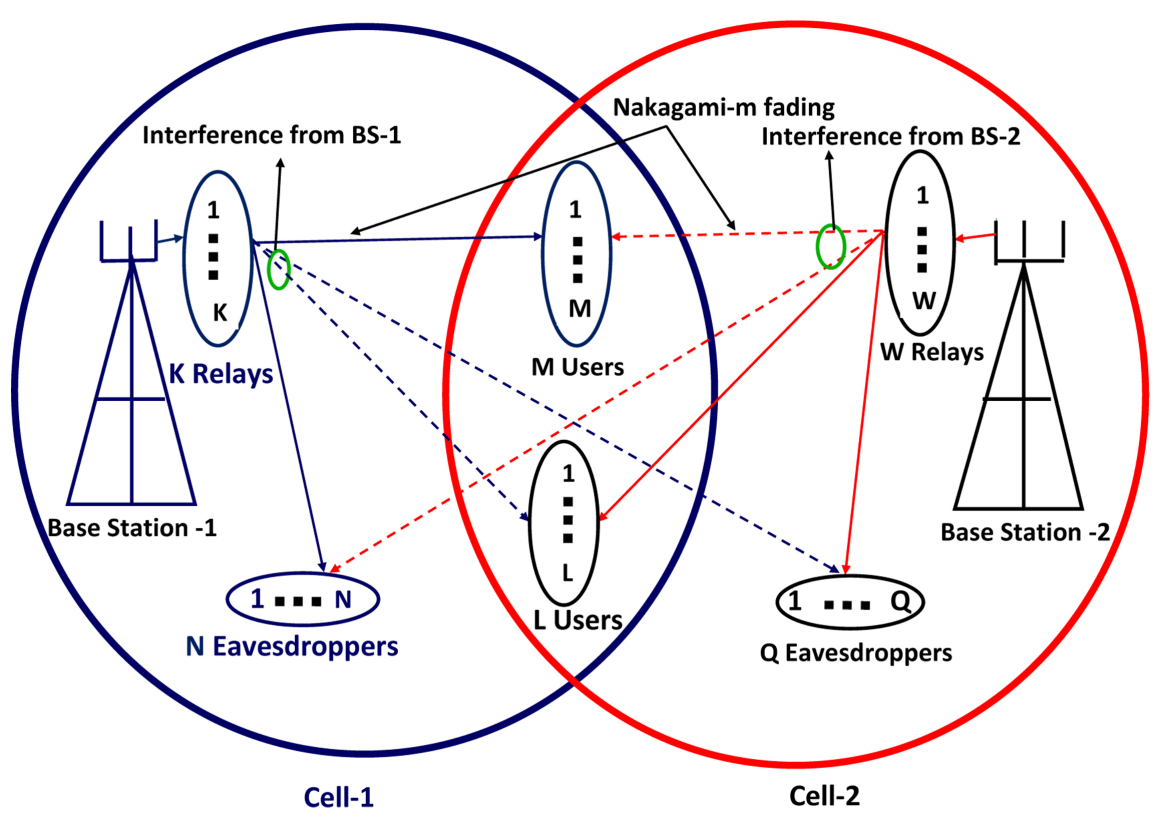

Figure 1. System model. 
It is assumed that there are no direct paths from base stations to destinations and eavesdroppers in Cell-1 and Cell-2, and communication occurs only through the relays. In the case of Cell-1, BS- 1 transmits $x_{1}$ symbol to $K$ relays in the first-hop. Therefore, received signal at the $k$ th relay denoted by $r_{k}$ is given by,

$$
r_{k}=h_{s, k} x_{1}+\mu_{k}
$$

where $h_{s, k}$ denotes the channel coefficient between BS- 1 and $k$ th relay and $\mu_{k} \sim \tilde{\mathcal{N}}\left(0, \lambda_{1}\right)$ denotes the additive white Gaussian noise at the $k$ th relay with zero and variance $\lambda_{1}$. In the second hop, at first, the selection of the best relay (i.e. the relay with the highest SNR) is performed using order statistics, so that only the best relay, $R_{b}$ forwards message to the $M$ destination users. During the transmission of information from BS-1 to destination user via relays, $N$ eavesdroppers try to decode the transmitted information.

Let $\mathbf{y}_{d_{i}}$ and $\mathbf{y}_{e_{j}}$ denote the received signals at the $i$ th destination and $t$ th eavesdropper, respectively. The channel gains from the best relay of Cell-1 to $i$ th $(i=1, \cdots, M)$ destination user and best relay of Cell-1 to $j$ th $(j=1, \cdots, N)$ eavesdropper are denoted by $\mathbf{h}_{b, i} \in \mathbb{C}^{n_{1 \times 1}}$ and $\mathbf{u}_{b, j} \in \mathbb{C}^{n_{e \times 1}}$, respectively. Without considering interference from Cell-2, we have

$$
\begin{aligned}
\mathbf{y}_{d_{i}} & =A_{k} \mathbf{h}_{b, i} r_{k}+\mathbf{w}_{m, i}=A_{k} \mathbf{h}_{b, i}\left(h_{s, k} x_{1}+\mu_{k}\right)+\mathbf{w}_{m, i} \\
& =A_{k} \mathbf{h}_{b, i} h_{s, k} x_{1}+A_{k} \mathbf{h}_{b, i} \mu_{k}+\mathbf{w}_{m, i}=A_{k} \mathbf{h}_{i}+\mathbf{a}_{m, i}, \\
\mathbf{y}_{e_{j}} & =A_{k} \mathbf{u}_{b, j} r_{k}+\mathbf{w}_{e, i}=A_{k} \mathbf{u}_{b, j}\left(h_{s, k} x_{1}+\mu_{k}\right)+\mathbf{w}_{e, i} \\
& =A_{k} \mathbf{u}_{b, j} h_{s, k} x_{1}+A_{k} \mathbf{u}_{b, j} \mu_{k}+\mathbf{w}_{e, i}=A_{k} \mathbf{u}_{j}+\mathbf{a}_{e, i},
\end{aligned}
$$

where $A_{k}=\frac{1}{\sqrt{E_{s}\left|h_{s, k}\right|^{2}+\lambda_{1}}}$ is the scaling factor at the $k$ th relay. $E_{s}$ is the average power per symbol. $\mathbf{h}_{i} \triangleq \mathbf{h}_{b, i} h_{s, k}$ and $\mathbf{u}_{j} \triangleq \mathbf{u}_{b, j} h_{s, k}$ are the channel coefficients from the best relay of Cell-1 to $i$ th destination and $j$ th eavesdropper, respectively. $\mathbf{a}_{m, i} \triangleq A_{k} \mathbf{h}_{b, i} \mu_{k}+\mathbf{w}_{m, i}$ and $\mathbf{a}_{e, i} \triangleq A_{k} \mathbf{u}_{b, j} \mu_{k}+\mathbf{w}_{e, i}$, where $\mathbf{w}_{m, i} \sim \mathcal{C} \tilde{\mathcal{N}}\left(0, \delta_{m}\right)$ and $\mathbf{w}_{e, i} \sim \mathcal{C} \tilde{\mathcal{N}}\left(0, \delta_{e}\right)$ denote the complex additive white Gaussian noises with zero mean and variances $\delta_{m}$ and $\delta_{e}$ imposed on the receiver at the $i$ th destination and th eavesdropper, respectively.

In the case of Cell-2, BS-2 transmits $x_{2}$ symbol to $W$ relays in the first-hop. Therefore, received signal at the $u$ th relay denoted by $r_{w}$ is given by,

$$
r_{w}=h_{s, w} x_{2}+v_{w}
$$

where $h_{s, w}$ denotes the channel coefficient between BS-2 and $w$ th relay and $\mu_{w} \sim \tilde{\mathcal{N}}\left(0, \lambda_{2}\right)$ denotes the additive white Gaussian noise at the $u$ th relay with zero and variance $\lambda_{2}$. In the second hop, at first, the selection of the best relay is performed using order statistics, so that only the best relay, $R_{b}$ forwards message to the $L$ destination users. During the transmission of information from BS-2 to destination user via relays, $Q$ eavesdroppers try to decode the transmitted information. 
Let $\mathbf{z}_{d_{l}}$ and $\mathbf{z}_{e_{q}}$ denote the received signals at the th destination and $q$ th eavesdropper, respectively. The channel gains from the best relay of Cell-2 to th $(l=1, \cdots, L)$ destination user and best relay of Cell-2 to $q$ th $(q=1, \cdots, Q)$ eavesdropper are denoted by $\mathbf{g}_{b, l} \in \mathbb{C}^{n_{12 \times 1}}$ and $\mathbf{v}_{b, q} \in \mathbb{C}^{n_{e_{2} \times 1}}$, respectively. Without considering interference from Cell-1, we have

$$
\begin{aligned}
\mathbf{z}_{d_{l}} & =B_{w} \mathbf{g}_{b, l} r_{w}+\mathbf{w}_{m, l}=B_{w} \mathbf{g}_{b, l}\left(h_{s, w} x_{2}+v_{w}\right)+\mathbf{w}_{m, l} \\
& =B_{w} \mathbf{g}_{b, l} h_{s, w} x_{2}+B_{w} \mathbf{g}_{b, l} v_{w}+\mathbf{w}_{m, l}=B_{w} \mathbf{g}_{l}+\mathbf{a}_{m, l}, \\
\mathbf{z}_{e_{q}} & =B_{w} \mathbf{v}_{b, q} r_{w}+\mathbf{w}_{e, q}=B_{w} \mathbf{v}_{b, q}\left(h_{s, w} x_{2}+v_{w}\right)+\mathbf{w}_{e, q} \\
= & B_{w} \mathbf{v}_{b, q} h_{s, w} x_{2}+B_{w} \mathbf{v}_{b, q} v_{w}+\mathbf{w}_{e, q}=B_{w} \mathbf{v}_{q}+\mathbf{a}_{m, q},
\end{aligned}
$$

where $B_{w}=\frac{1}{\sqrt{E_{s}\left|h_{s, w}\right|^{2}+\lambda_{2}}}$ is the scaling factor at the $w$ th relay. $\mathbf{g}_{l} \triangleq \mathbf{g}_{b, l} h_{s, w}$ and $\mathbf{v}_{q} \triangleq \mathbf{v}_{b, q} h_{s, w}$ are the channel coefficients from the best relay of Cell-2 to th destination and $q$ th eavesdropper, respectively. $\mathbf{a}_{m, l} \triangleq B_{w} \mathbf{g}_{b, l} v_{w}+\mathbf{w}_{m, l}$ and $\mathbf{a}_{m, q} \triangleq B_{w} \mathbf{v}_{b, q} v_{w}+\mathbf{w}_{e, q}$, where $\mathbf{w}_{m, l} \sim \mathcal{C} \tilde{\mathcal{N}}\left(0, \kappa_{m}\right)$ and $\mathbf{w}_{e, q} \sim \mathcal{C N}\left(0, \kappa_{e}\right)$ denote the complex additive white Gaussian noises with zero mean and variances $\kappa_{m}$ and $\kappa_{e}$ imposed on the receiver at the th destination and $q$ th eavesdropper, respectively.

Let, $\mathbf{g}_{b, i} \in \mathbb{C}^{n_{1 \times 1}}$ and $\mathbf{v}_{b, j} \in \mathbb{C}^{n_{e \times 1}}$ channel gain from the best relay of Cell-2 to $t$ th user and to th eavesdropper of Cell-1. $\mathbf{h}_{b, l} \in \mathbb{C}^{n_{12 \times 1}}$ and $\mathbf{u}_{b, q} \in \mathbb{C}^{n_{e_{2} \times 1}}$ channel gain from the best relay of Cell-1 to th user and to $q$ th eavesdropper of Cell-2. Then, considering interference from Cell-2, received signal at the ith user and the $j$ th eavesdropper of Cell- 1 are given by,

$$
\begin{array}{r}
\mathbf{y}_{d_{i}}^{c}=A_{k} \mathbf{h}_{i}+\mathbf{g}_{b, i} r_{w}+\mathbf{a}_{m, i}=A_{k} \mathbf{h}_{i}+\mathbf{g}_{b, i}\left(h_{s, w} x_{2}+v_{w}\right)+\mathbf{a}_{m, i}=A_{k} \mathbf{h}_{i}+\Psi_{i} \\
\mathbf{y}_{e_{j}}^{c}=A_{k} \mathbf{u}_{j}+\mathbf{v}_{b, j} r_{w}+\mathbf{a}_{e, i}=A_{k} \mathbf{u}_{j}+\mathbf{v}_{b, j}\left(h_{s, w} x_{2}+v_{w}\right)+\mathbf{a}_{e, i}=A_{k} \mathbf{u}_{j}+\Phi_{j},
\end{array}
$$

where $\Psi_{i} \triangleq \mathbf{g}_{b, i}\left(h_{s, w} x_{2}+v_{w}\right)+\mathbf{a}_{m, i} \in \mathcal{C} \tilde{\mathcal{N}}\left(0, I_{m_{1}}\right)$ denotes the interference plus noise vector at the $i$ th user of Cell-1 with zero mean and variance $I_{m_{1}}$. Similarly, $\Phi_{j} \triangleq \mathbf{v}_{b, j}\left(h_{s, w} x_{2}+v_{w}\right)+\mathbf{a}_{e, i} \in \mathcal{C} \tilde{\mathcal{N}}\left(0, I_{e_{1}}\right)$ denotes the interference plus noise vector at the $j$ th eavesdropper of Cell-1 with zero mean and variance $I_{e_{1}}$. Similarly, considering interference from Cell-1, received signal at the th user and the $q$ th eavesdropper of Cell-2 are given by,

$$
\begin{gathered}
\mathbf{z}_{d_{l}}^{c}=B_{w} \mathbf{g}_{l}+\mathbf{h}_{b, l} r_{k}+\mathbf{a}_{m, l}=B_{w} \mathbf{g}_{l}+\mathbf{h}_{b, l}\left(h_{s, k} x_{2}+\mu_{k}\right)+\mathbf{a}_{m, l}=B_{w} \mathbf{g}_{l}+\Upsilon_{l} \\
\mathbf{z}_{e_{q}}^{c}=B_{w} \mathbf{v}_{q}+\mathbf{u}_{b, q} r_{k}+\mathbf{a}_{e, q}=B_{w} \mathbf{v}_{q}+\mathbf{u}_{b, q}\left(h_{s, k} x_{2}+\mu_{k}\right)+\mathbf{a}_{e, q}=B_{w} \mathbf{v}_{q}+\Xi_{q},
\end{gathered}
$$

where $\Upsilon_{l} \triangleq \mathbf{h}_{b, l}\left(h_{s, k} x_{2}+\mu_{k}\right)+\mathbf{a}_{m, l} \in \mathcal{C} \tilde{\mathcal{N}}\left(0, I_{m_{2}}\right)$ denotes the interference plus noise vector at the th user of Cell-2 with zero mean and variance $I_{m_{2}}$. Similarly, $\Xi_{q} \triangleq \mathbf{u}_{b, q}\left(h_{s, k} x_{2}+\mu_{k}\right)+\mathbf{a}_{e, q} \in \mathcal{C N} \tilde{\mathcal{N}}\left(0, I_{e_{2}}\right)$ denotes the interference plus noise vector at the $q$ th eavesdropper of Cell-2 with zero mean and variance $I_{e_{2}}$.

In the case of Cell-1, the instantaneous SINRs at the $k$ th relay, the th destination and th eavesdropper can be expressed as 


$$
\begin{gathered}
\gamma_{s, k}=\bar{\gamma}_{o 1}\left|h_{s, k}\right|^{2}, \\
\gamma_{k, i}=\frac{\left|\mathbf{h}_{i}\right|^{2}}{\sqrt{\left|\mathbf{g}_{b, i} h_{s, w}\right|^{2}+\left|\mathbf{g}_{b, i}\right|^{2}+\left|\mathbf{a}_{m, i}\right|^{2}}}, \\
\gamma_{k, j}=\frac{\left|\mathbf{u}_{j}\right|^{2}}{\sqrt{\left|\mathbf{v}_{b, j} h_{s, w}\right|^{2}+\left|\mathbf{v}_{b, j}\right|^{2}+\left|\mathbf{a}_{e, i}\right|^{2}}},
\end{gathered}
$$

where $\bar{\gamma}_{o 1}$ denotes the average SNR of the first hop for Cell-1. Similarly, in the case of Cell-2, the instantaneous SINRs at the $w$ th relay, the th destination and $q$ th eavesdropper can be expressed as

$$
\begin{gathered}
\gamma_{s, w}=\bar{\gamma}_{o 2}\left|h_{s, w}\right|^{2}, \\
\gamma_{w, l}=\frac{\left|\mathbf{g}_{l}\right|^{2}}{\sqrt{\left|\mathbf{h}_{b, l} h_{s, k}\right|^{2}+\left|\mathbf{h}_{b, l}\right|^{2}+\left|\mathbf{a}_{m, l}\right|^{2}}}, \\
\gamma_{w, q}=\frac{\left|\mathbf{v}_{q}\right|^{2}}{\sqrt{\left|\mathbf{u}_{b, q} h_{s, w}\right|^{2}+\left|\mathbf{u}_{b, q}\right|^{2}+\left|\mathbf{a}_{e, q}\right|^{2}}},
\end{gathered}
$$

where $\bar{\gamma}_{o 2}$ denotes the average SNR of the first hop for Cell-2.

\section{Problem Formulation}

Since the communication is performed via best relay, therefore its selection is an important issue to develop the mathematical model which ensures the security of proposed model. The selection of the best relay is performed in two steps by order statistics. In the first step, for each relay node, the weaker links between the first hop and the second hop are searched for applying order statistics. In the second step, the relay having largest equivalent end-to-end SINRs are selected as the candidate relay to perform detection, amplification and forward the message. Therefore, in the case of Cell-1, based on the links strength, the equivalent end-to-end SINRs at the $k$ th relay for the destination links, $\gamma_{e q_{k}}$ and for the eavesdropper links, $\beta_{e q_{k}}$ are ordered as follows [19] [20]:

$$
\begin{aligned}
& \gamma_{e q_{k}}=\min \left\{\gamma_{s, k}, \gamma_{k, i}\right\} \\
& \beta_{e q_{k}}=\min \left\{\gamma_{s, k}, \gamma_{k, j}\right\}
\end{aligned}
$$

The instantaneous SINRs at the output of the relay selection combiner for ith destination and th eavesdropper can be expressed, respectively as

$$
\begin{aligned}
& \gamma_{\Sigma_{d_{i}}}=\max _{k=1,2, \cdots, K} \delta_{e q_{w}} \\
& \beta_{\Sigma_{e_{j}}}=\max _{k=1,2, \cdots, K} \beta_{e q_{k}}
\end{aligned}
$$

The probability density function (PDF) of SINRs, $\gamma_{s, k}, \gamma_{k, i}$ and $\gamma_{k, j}$ for correlated Nakagami- $m$ fading channel are respectively given by [21] . 


$$
\begin{gathered}
f\left(\gamma_{s, k}\right) \approx \frac{\gamma_{s, k}^{\bar{m}_{1 k}-1} \mathrm{e}^{-\frac{\bar{m}_{1 k} \gamma_{s, k}}{\bar{\Omega}_{1 k}}}}{\left(\frac{\bar{\Omega}_{1 k}}{\bar{m}_{1 k}}\right)^{\bar{m}_{1 k}} \Gamma\left(\bar{m}_{1 k}\right)}=a_{1} \frac{\gamma_{s, k}^{\bar{m}_{1 k}-1}}{\mathrm{e}^{\frac{\bar{m}_{k} \gamma_{s, k}}{\bar{\Omega}_{1 k}}}, \gamma_{s, k} \geq 0} \\
f\left(\gamma_{k, i}\right) \approx \frac{\gamma_{k, i}^{\bar{m}_{1 i}-1} \mathrm{e}^{-\frac{\bar{m}_{1 i} \gamma_{k, i}}{\bar{\Omega}_{1 i}}}}{\left(\frac{\bar{\Omega}_{1 i}}{\bar{m}_{1 i}}\right)^{\bar{m}_{1 i}} \Gamma\left(\bar{m}_{1 i}\right)}=a_{2} \frac{\gamma_{k, i}^{\bar{m}_{1 i}-1}}{\mathrm{e}^{\bar{m}_{1 i} \gamma_{k, i}}}, \gamma_{k, i} \geq 0 \\
f\left(\gamma_{k, j}\right) \approx \frac{\gamma_{k, j}^{\bar{m}_{1 i}-1} \mathrm{e}^{-\frac{\bar{m}_{1 j} \gamma_{k, j}}{\bar{\Omega}_{1 j}}}}{\left(\frac{\bar{\Omega}_{1 j}}{\bar{m}_{1 j}}\right)^{\bar{m}_{1 j}} \Gamma\left(\bar{m}_{1 j}\right)}=a_{3} \frac{\gamma^{\frac{\gamma_{k, j}}{\bar{m}_{1 j}-1}}}{\mathrm{e}^{\bar{\Omega}_{1 j}}}, \gamma_{k, j} \geq 0
\end{gathered}
$$

where $m$ denotes the Nakagami- $m$ fading parameter. $a_{1}=\frac{1}{\left(\frac{\bar{\Omega}_{1 k}}{\bar{m}_{1 k}}\right)^{\bar{m}_{1 k}} \Gamma\left(\bar{m}_{1 k}\right)}$,

$\bar{m}_{1 k}=\frac{m}{\zeta\left(1, \bar{\kappa}_{m 1}\right)}, \zeta\left(1, \bar{\kappa}_{m 1}\right)=1+\frac{2 \bar{\kappa}_{m 1}}{1-\bar{\kappa}_{m 1}}\left(1-\frac{1-\bar{\kappa}_{m 1}}{1-\bar{\kappa}_{m 1}}\right), \bar{\kappa}_{m 1}=\rho_{m_{1}}^{2}$ and

$\bar{\Omega}_{1 k}=\mathbb{E}\left\{\left\|h_{s, k}\right\|^{2}\right\} \cdot a_{2}=\frac{1}{\left(\frac{\bar{\Omega}_{1 i}}{\bar{m}_{1 i}}\right)^{\bar{m}_{1 i}} \Gamma\left(\bar{m}_{1 i}\right)}, \quad \bar{m}_{1 i}=\frac{n_{r_{1}}^{2} m}{\zeta\left(n_{r_{1}}, \bar{\kappa}_{m 1}\right)}$,

$\zeta\left(n_{\eta_{1}}, \bar{\kappa}_{m 1}\right)=n_{\eta_{1}}+\frac{2 \bar{\kappa}_{m 1}}{1-\bar{\kappa}_{m 1}}\left(n_{\eta}-\frac{1-\bar{\kappa}_{m 1}^{n_{\eta 1}}}{1-\bar{\kappa}_{m 1}}\right), \bar{\kappa}_{m 1}=\rho_{m_{1}}^{2}$ and $\bar{\Omega}_{1 i}=\mathbb{E}\left\{\left\|\mathbf{h}_{i}\right\|^{2}\right\}$.

$a_{3}=\frac{1}{\left(\frac{\bar{\Omega}_{1 j}}{\bar{m}_{1 j}}\right)^{\bar{m}_{1 j}} \Gamma\left(\bar{m}_{1 j}\right)}, \bar{m}_{1 j}=\frac{n_{e_{1}}^{2} m}{\zeta\left(n_{e_{1}}, \bar{\kappa}_{m 2}\right)}$,

$\zeta\left(n_{e_{1}}, \bar{\kappa}_{m 2}\right)=n_{e_{1}}+\frac{2 \bar{\kappa}_{m 2}}{1-\bar{\kappa}_{m 2}}\left(n_{e_{1}}-\frac{1-\bar{\kappa}_{m 2}^{n_{e 1}}}{1-\bar{\kappa}_{m 2}}\right), \bar{\kappa}_{m 2}=\rho_{e_{1}}^{2}$ and $\bar{\Omega}_{1 j}=\mathbb{E}\left\{\left\|\mathbf{u}_{j}\right\|^{2}\right\}$.

In the case of Cell-2, based on the links strength, the equivalent end-to-end SINRs at the $w$ th relay for the destination links, $\delta_{e q_{w}}$ and for the eavesdropper links, $\xi_{e q_{w}}$ are ordered as follows [19] [20]:

$$
\begin{aligned}
& \delta_{e q_{w}}=\min \left\{\gamma_{s, w}, \gamma_{w, l}\right\} \\
& \xi_{e q_{w}}=\min \left\{\gamma_{s, w}, \gamma_{w, q}\right\}
\end{aligned}
$$

The instantaneous SINRs at the output of the relay selection combiner for th destination and $q$ th eavesdropper can be expressed, respectively as

$$
\begin{aligned}
& \delta_{\Sigma_{d_{l}}}=\max _{w=1,2, \cdots, W} \gamma_{e q_{k}} \\
& \xi_{\Sigma_{e_{q}}}=\max _{w=1,2, \cdots, W} \xi_{e q_{w}}
\end{aligned}
$$

The PDFs of SINRs, $\gamma_{s, w}, \gamma_{w, l}$ and $\gamma_{w, q}$ for correlated Nakagami- $m$ fading channel are respectively given by [21]. 


$$
\begin{gathered}
f\left(\gamma_{s, w}\right) \approx \frac{\gamma_{s, w}^{\bar{m}_{1 w}-1} \mathrm{e}^{-\frac{\bar{m}_{1 w} \gamma_{s, w}}{\bar{\Omega}_{1 w}}}}{\left(\frac{\bar{\Omega}_{1 w}}{\bar{m}_{1 w}}\right)^{\bar{m}_{1 w}} \Gamma\left(\bar{m}_{1 w}\right)}=b_{1} \frac{\gamma_{s, w}^{\bar{m}_{1 w}-1}}{\mathrm{e}^{\frac{\bar{m}_{1 w} \gamma_{s, w}}{\bar{\Omega}_{1 w}}}, \gamma_{s, w} \geq 0} \\
f\left(\gamma_{w, l}\right) \approx \frac{\gamma_{w, l}^{\bar{m}_{1, l}-1} \mathrm{e}^{-\frac{\bar{m}_{1 l} \gamma_{w, l}}{\bar{\Omega}_{1 l}}}}{\left(\frac{\bar{\Omega}_{1 l}}{\bar{m}_{1 l}}\right)^{\bar{m}_{1 l}} \Gamma\left(\bar{m}_{1 l}\right)}=b_{2} \frac{\gamma_{w, l}^{\bar{m}_{1 l}-1}}{\mathrm{e}^{\bar{m}_{1 l} \gamma_{w, l}}}, \gamma_{w, l} \geq 0 \\
f\left(\gamma_{w, q}\right) \approx \frac{\gamma_{w, q}^{\bar{\Omega}_{1 l}-1} \mathrm{e}^{-\frac{\bar{m}_{1 q} \gamma_{w, q}}{\bar{\Omega}_{1 q}}}}{\left(\frac{\bar{\Omega}_{1 q}}{\bar{m}_{1 q}}\right)^{\bar{m}_{1 q}} \Gamma\left(\bar{m}_{1 q}\right)}=b_{3} \frac{\mathrm{\gamma}^{\bar{m}_{w, q}}}{\mathrm{~m}_{1 q}^{\bar{m}_{1 q}-1}}, \gamma_{w, q} \geq 0
\end{gathered}
$$

where $b_{1}=\frac{1}{\left(\frac{\bar{\Omega}_{1 w}}{\bar{m}_{1 w}}\right)^{\bar{m}_{1 w}} \Gamma\left(\bar{m}_{1 w}\right)}, \quad \bar{m}_{1 w}=\frac{m}{\zeta\left(1, \bar{\kappa}_{m 2}\right)}$,

$\zeta\left(1, \bar{\kappa}_{m 2}\right)=1+\frac{2 \bar{\kappa}_{m 2}}{1-\bar{\kappa}_{m 2}}\left(1-\frac{1-\bar{\kappa}_{m 2}}{1-\bar{\kappa}_{m 2}}\right), \quad \bar{\kappa}_{m 2}=\rho_{m_{2}}^{2}$ and $\bar{\Omega}_{1 w}=\mathbb{E}\left\{\left\|h_{s, w}\right\|^{2}\right\}$.

$b_{2}=\frac{1}{\left(\frac{\bar{\Omega}_{1 l}}{\bar{m}_{1 l}}\right)^{\bar{m}_{1 l}} \Gamma\left(\bar{m}_{1 l}\right)}, \quad \bar{m}_{1 l}=\frac{n_{r_{2}}^{2} m}{\zeta\left(n_{r_{2}}, \bar{\kappa}_{m 2}\right)}$,

$\zeta\left(n_{r_{2}}, \bar{\kappa}_{m 2}\right)=n_{r_{2}}+\frac{2 \bar{\kappa}_{m 2}}{1-\bar{\kappa}_{m 2}}\left(n_{r_{2}}-\frac{1-\bar{\kappa}_{m 2}^{n_{l_{2}}}}{1-\bar{\kappa}_{m 2}}\right), \bar{\kappa}_{m 2}=\rho_{m_{2}}^{2}$ and $\bar{\Omega}_{1 l}=\mathbb{E}\left\{\left\|\mathbf{g}_{l}\right\|^{2}\right\}$.

$b_{3}=\frac{1}{\left(\frac{\bar{\Omega}_{1 q}}{\bar{m}_{1 q}}\right)^{\bar{m}_{1 q}} \Gamma\left(\bar{m}_{1 q}\right)}, \bar{m}_{1 q}=\frac{n_{e_{q}}^{2} m}{\zeta\left(n_{e_{q}}, \bar{\kappa}_{e 2}\right)}$,

$\zeta\left(n_{e_{2}}, \bar{\kappa}_{e 2}\right)=n_{e_{2}}+\frac{2 \bar{\kappa}_{e 2}}{1-\bar{\kappa}_{e 2}}\left(n_{e_{2}}-\frac{1-\bar{\kappa}_{e 2}^{n_{e 2}}}{1-\bar{\kappa}_{e 2}}\right), \bar{\kappa}_{e 2}=\rho_{e_{2}}^{2}$ and $\bar{\Omega}_{1 q}=\mathbb{E}\left\{\left\|\mathbf{v}_{q}\right\|^{2}\right\}$.

\section{Best Relay Selection for Secure Multicasting}

In order to derive the analytical expressions for the probability of non-zero secrecy multicast capacity and the secure outage probability for multicasting, we need the PDF of the SINRs for the best relay, multicast as well as eavesdropper channels, the formulation of which are illustrated as follows [22]:

1) The PDF of SINR for the Best Relay: In the case of Cell-1, let $F_{\gamma_{\text {eq }}}\left(\gamma_{i}\right)$ denotes the cumulative distribution function of $\gamma_{e q_{k}}$, then we have

$$
F_{\gamma_{\text {eqk }}}\left(\gamma_{i}\right)=1-\operatorname{Pr}\left(\gamma_{s, k}>\gamma_{i}\right) \operatorname{Pr}\left(\gamma_{k, i}>\gamma_{i}\right) \text {. }
$$

Now, $\operatorname{Pr}\left(\gamma_{s, k}>\gamma_{i}\right)$ can be defined as

$$
\operatorname{Pr}\left(\gamma_{s, k}>\gamma_{i}\right) \triangleq \int_{\gamma_{i}}^{\infty} f\left(\gamma_{s, k}\right) \mathrm{d} \gamma_{s, k}=\int_{\gamma_{i}}^{\infty} a_{1} \gamma_{s, k}^{\bar{m}_{1 k}-1} \mathrm{e}^{-\frac{\bar{m}_{1 k} \gamma_{s, k}}{\bar{\Omega}_{1 k}}} \mathrm{~d} \gamma_{s, k}
$$


Using the following identity of [23] stated in equation (3.351.2),

$$
\int_{u}^{\infty} x^{n} \mathrm{e}^{-\mu x} \mathrm{~d} x=\mathrm{e}^{-\mu u} \sum_{k=0}^{n}\left(\frac{n ! u^{k}}{k ! \mu^{n-k+1}}\right)
$$

we have

$$
\operatorname{Pr}\left(\gamma_{s, k}>\gamma_{i}\right)=a_{1} \sum_{k_{1}}^{\bar{m}_{1 k}-1} B_{1} \gamma_{i}^{k_{1}+1} \mathrm{e}^{-\frac{\bar{m}_{\bar{l}_{1 k}}}{\bar{\Omega}_{1 k}}}
$$

where $B_{1}=\frac{\left(\bar{m}_{1 k}-1\right) !}{k_{1} !\left(\frac{\bar{m}_{1 k}}{\bar{\Omega}_{1 k}}\right)^{\bar{m}_{1 k}-k_{1}}} \cdot$ Similarly,

$$
\operatorname{Pr}\left(\gamma_{k, i}>\gamma_{i}\right)=a_{2} \sum_{k_{2}}^{\bar{m}_{1 i}-1} D_{1} \gamma_{i}^{k_{2}+1} \mathrm{e}^{-\frac{\bar{m}_{1 i}}{\bar{s}_{1 i}}}
$$

where $D_{1}=\frac{\left(\bar{m}_{1 i}-1\right) !}{k_{2} !\left(\frac{\bar{m}_{1 i}}{\bar{\Omega}_{1 i}}\right)^{\bar{m}_{1 i}-k_{2}}}$. Substituting the values of $\operatorname{Pr}\left(\gamma_{s, k}>\gamma_{i}\right)$ and

$\operatorname{Pr}\left(\gamma_{k, i}>\gamma_{i}\right)$ in Equation (19), we have

$$
F_{\gamma_{\text {eq }}}\left(\gamma_{i}\right)=1-a_{1} a_{2} \sum_{k_{1}=0}^{\bar{m}_{1 k}-1} \sum_{k_{2}=0}^{\bar{m}_{i i}-1} B_{1} D_{1} \frac{\gamma_{i}^{k_{1}+k_{2}}}{\mathrm{e}^{\frac{m_{k i} \gamma_{i}}{\bar{\Omega}_{k i}}}}
$$

where $\bar{m}_{k i}=\bar{m}_{1 k}+\bar{m}_{1 i}$ and $\bar{\Omega}_{k i}=\bar{\Omega}_{1 k}+\bar{\Omega}_{1 i}$. Differentiating Equation (24), the PDF of $\gamma_{e q_{k}}$ can be obtained as follows;

$$
\begin{aligned}
& f_{\gamma_{\text {eq }}}\left(\gamma_{i}\right)=\frac{\mathrm{d}}{\mathrm{d} \gamma_{i}}\left\{F_{\gamma_{\text {eqk }}}\left(\gamma_{i}\right)\right\} \\
& =-a_{1} a_{2} \sum_{k_{1}=0}^{\bar{m}_{1 k}-1} \sum_{k_{2}=0}^{\bar{m}_{1 i}-1} B_{1} D_{1}\left\{\gamma_{i}^{k_{1}+k_{2}}\left(-\frac{\bar{m}_{k i}}{\bar{\Omega}_{k i}}\right) \mathrm{e}^{-\frac{\bar{m}_{k i} \gamma_{i}}{\bar{\Omega}_{k i}}}+\mathrm{e}^{-\frac{\bar{m}_{k i} \gamma_{i}}{\bar{\Omega}_{k i}}}\left(k_{1}+k_{2}\right) \gamma_{i}^{k_{1}+k_{2}-1}\right\}
\end{aligned}
$$

Therefore, the PDF of $\gamma_{\Sigma_{d_{i}}}$ is defined as

$$
f_{\gamma_{\Sigma_{d_{i}}}}\left(\gamma_{i}\right)=K f_{\gamma_{\text {eqk }}}\left(\gamma_{i}\right)\left[F_{\text {eqk }}\left(\gamma_{i}\right)\right]^{K-1}
$$

Substituting Equations (24) and (25) into (26), and using the following identity of [23] stated in equation (1.111),

$$
(a+x)^{n}=\sum_{i=0}^{n}\left(\begin{array}{l}
n \\
i
\end{array}\right) x^{i} a^{n-i},
$$

the PDF of $\gamma_{\Sigma_{d_{i}}}$ is given by

$$
f_{\gamma_{\Sigma_{d_{i}}}}\left(\gamma_{i}\right)=K Q_{m_{1}} R_{m_{1}}\left[\bar{m}_{k i}\left\{\frac{\gamma_{i}^{\alpha_{1}}}{\bar{\Omega}_{k i}}\left\{\mathrm{e}^{\frac{\bar{m}_{k i}\left(l_{1}+1\right) \gamma_{i}}{\bar{\Omega}_{k i}}}\right\}-\left(k_{1}+k_{2}\right)\left\{\frac{\gamma_{i}^{\alpha_{1}-\left(k_{1}+k_{2}\right) \gamma_{1}}}{\mathrm{e}^{\frac{\bar{m}_{k i}\left(l_{1}+1\right) \gamma_{i}}{\bar{\Omega}_{k i}}}}\right\}\right],\right.
$$

where $Q_{m_{1}}=a_{1} a_{2} \sum_{k_{1}=0}^{\bar{m}_{1 k}-1} \sum_{k_{2}=0}^{\bar{m}_{1 k}-1} B_{1} D_{1}$,

$$
R_{m_{1}}=\sum_{l_{1}=0}^{k_{1}-1}\left(\begin{array}{c}
k_{1}-1 \\
l_{1}
\end{array}\right)(-1)^{l_{1}}\left(a_{1} a_{2}\right)^{l_{1}} \sum_{k_{1}=0}^{l_{1}\left(\bar{m}_{1 k}-1\right)}\left(B_{1} D_{1}\right)^{l_{1}} \text { and } \alpha_{1}=\left(k_{1}+k_{2}\right)\left(l_{1}+1\right) \text {. }
$$


Following the similar procedure of $f_{\gamma_{\Sigma_{d_{i}}}}\left(\gamma_{i}\right)$, the PDF of $\beta_{\Sigma_{e_{j}}}$ denoted by $f_{\beta_{\Sigma_{e_{j}}}}\left(\gamma_{j}\right)$ can be derived as

$$
f_{\beta_{\Sigma_{e_{j}}}}\left(\gamma_{j}\right)=K Q_{e_{1}} R_{e_{1}}\left[\frac{\bar{m}_{k j}}{\bar{\Omega}_{k j}}\left\{\frac{\gamma_{j}^{\lambda_{1}}}{\mathrm{e}^{\frac{\bar{m}_{k j}\left(l_{1}+1\right) \gamma_{j}}{\bar{\Omega}_{k j}}}}\right\}-\left(k_{1}+k_{2}\right)\left\{\frac{\gamma_{j}^{\lambda_{1}-\left(k_{1}+k_{3}\right) \gamma_{1}}}{\mathrm{e}^{\frac{\bar{m}_{k j}\left(l_{1}+1\right) \gamma_{j}}{\bar{\Omega}_{k j}}}}\right\}\right],
$$

where $Q_{\ell_{1}}=a_{1} a_{3} \sum_{k_{1}=0}^{\bar{m}_{1 k}-1} \sum_{k_{3}=0}^{\bar{m}_{1 j}-1} B_{1} G_{1}$,

$$
\begin{aligned}
& R_{e_{1}}=\sum_{l_{1}=0}^{k_{1}-1}\left(\begin{array}{c}
k_{1}-1 \\
l_{1}
\end{array}\right)(-1)^{l_{1}}\left(a_{1} a_{3}\right)^{l_{1}} \sum_{k_{1}=0}^{l_{1}\left(\bar{m}_{1 k}-1\right)}\left(B_{1} G_{1}\right)^{l_{1}}, \bar{m}_{k j}=\bar{m}_{1 k}+\bar{m}_{1 j}, \\
& \left.\bar{\Omega}_{k j}=\bar{\Omega}_{1 k}+\bar{\Omega}_{1 j}, \quad \lambda_{1}=\left(k_{1}+k_{3}\right)\left(l_{1}+1\right) \text { and } G_{1}=\frac{\left(\bar{m}_{1 j}-1\right) !}{k_{3} !\left(\bar{m}_{1 j} \bar{\Omega}_{1 j}\right.}\right)^{\bar{m}_{1 j}-k_{3}}
\end{aligned}
$$

In the case of Cell-2, let $f_{\delta_{\Sigma_{l}}}\left(\gamma_{l}\right)$ and $f_{\xi_{\Sigma_{e_{q}}}}\left(\gamma_{q}\right)$ respectively denote the PDFs of $\delta_{\Sigma_{d_{l}}}$ and $\xi_{\Sigma_{e_{q}}}$. Following the similar procedure of $f_{\gamma_{\Sigma_{d_{i}}}}\left(\gamma_{i}\right)$, the expressions of $f_{\delta_{\Sigma_{d_{l}}}}\left(\gamma_{l}\right)$ and $f_{\xi_{\Sigma_{e_{q}}}}\left(\gamma_{q}\right)$ can be derived as

$$
f_{\gamma_{\Sigma_{d l}}}\left(\gamma_{l}\right)=W Q_{m_{2}} R_{m_{2}}\left[\frac{\bar{m}_{w l}}{\bar{\Omega}_{w l}}\left\{\frac{\gamma_{l}^{\alpha_{2}}}{\mathrm{e}^{\frac{\bar{m}_{w l}\left(\tau_{1}+1\right) \gamma_{l}}{\bar{\Omega}_{w l}}}}\right\}-\left(t_{1}+t_{2}\right)\left\{\frac{\gamma_{l}^{\alpha_{2}-\left(t_{1}+t_{2}\right) \tau_{1}}}{\mathrm{e}^{\frac{\bar{m}_{w l}\left(\tau_{1}+1\right) \gamma_{l}}{\bar{\Omega}_{w l}}}}\right\}\right],
$$

where $Q_{m_{2}}=b_{1} b_{2} \sum_{t_{1}=0}^{\bar{m}_{1}-1} \sum_{t_{2}=0}^{\bar{m}_{1}-1} B_{2} D_{2}$,

$$
\begin{aligned}
& R_{m_{2}}=\sum_{\tau_{1}=0}^{t_{1}-1}\left(\begin{array}{c}
t_{1}-1 \\
\tau_{1}
\end{array}\right)(-1)^{\tau_{1}}\left(b_{1} b_{2}\right)^{\tau_{1}} \sum_{t_{2}=0}^{\tau_{1}\left(\bar{m}_{1 w}-1\right)}\left(B_{2} D_{2}\right)^{\tau_{1}}, B_{2}=\frac{\left(\bar{m}_{1 w}-1\right) !}{t_{1} !\left(\frac{\bar{m}_{1 w}}{\bar{\Omega}_{1 w}}\right)^{\bar{m}_{1 w}-t_{1}}}, \\
& D_{2}=\frac{\left(\bar{m}_{1 l}-1\right) !}{t_{2} !\left(\frac{\bar{m}_{1 l}}{\bar{\Omega}_{1 l}}\right)^{\bar{m}_{1 l}-t_{2}}} \text { and } \alpha_{2}=\left(t_{1}+t_{2}\right)\left(\tau_{1}+1\right) . \\
& f_{\xi_{\Sigma_{e_{q}}}}\left(\gamma_{j}\right)=W Q_{e_{2}} R_{e_{2}}\left[\frac{\bar{m}_{w q}}{\bar{\Omega}_{w q}}\left\{\frac{\gamma_{q}^{\lambda_{2}}}{\mathrm{e}^{\frac{\bar{m}_{w q}\left(\tau_{1}+1\right) \gamma_{q}}{\bar{\Omega}_{w q}}}}\right\}-\left(t_{1}+t_{3}\right)\left\{\frac{\gamma_{q}^{\lambda_{2}-\left(t_{1}+t_{3}\right) \tau_{1}}}{\frac{\bar{m}_{w q}\left(\tau_{1}+1\right) \gamma_{q}}{\bar{\Omega}_{w q}}}\right\}\right],
\end{aligned}
$$

where $Q_{e_{2}}=b_{1} b_{3} \sum_{t_{1}=0}^{\bar{m}_{1 w}-1} \sum_{t_{3}=0}^{\bar{m}_{1 q}-1} B_{2} G_{2}$,

$$
\begin{aligned}
& R_{e_{2}}=\sum_{\tau_{1}=0}^{t_{1}-1}\left(\begin{array}{c}
t_{1}-1 \\
\tau_{1}
\end{array}\right)(-1)^{\tau_{1}}\left(b_{1} b_{3}\right)^{\tau_{1}} \sum_{t_{1}=0}^{\tau_{1}\left(\bar{m}_{1 w}-1\right)}\left(B_{2} G_{2}\right)^{\tau_{1}}, \bar{m}_{w q}=\bar{m}_{1 w}+\bar{m}_{1 q}, \\
& \bar{\Omega}_{w q}=\bar{\Omega}_{1 w}+\bar{\Omega}_{1 q}, \quad \lambda_{2}=\left(t_{1}+t_{3}\right)\left(\tau_{1}+1\right) \text { and } G_{2}=\frac{\left(\bar{m}_{1 q}-1\right) !}{t_{3} !\left(\frac{\bar{m}_{1 q}}{\bar{\Omega}_{1 q}}\right)^{\bar{m}_{1 q}-t_{3}}} .
\end{aligned}
$$

2) The PDF of SINR for Multicast Channels: In the case of Cell-1, let $d_{\text {min }} \triangleq \min _{1 \leq i \leq M} \gamma_{\Sigma_{d_{i}}}$, then the PDF of $d_{\text {min }}$ can be defined as [24] [25]

$$
f_{d_{\min }}\left(\gamma_{i}\right)=M f_{\gamma_{\Sigma_{d_{i}}}}\left(\gamma_{i}\right)\left[1-F_{\gamma_{\Sigma_{d_{i}}}}\left(\gamma_{i}\right)\right]^{M-1}=M f_{\gamma_{\Sigma_{d_{i}}}}\left(\gamma_{i}\right)\left[1-\int_{0}^{\gamma_{i}} f_{\gamma_{\Sigma_{d_{i}}}}\left(\gamma_{i}\right) \mathrm{d} \gamma_{i}\right]^{M-1}
$$


Substituting the values of $f_{\gamma_{\Sigma_{d_{i}}}}\left(\gamma_{i}\right)$ in Equation (32) and using the identity of Equation (27), we have

$$
f_{d_{\min }}\left(\gamma_{i}\right)=M K Q_{m_{1}} R_{m_{1}} T_{m_{1}}\left[\frac{\bar{m}_{k i}}{\bar{\Omega}_{k i}}\left\{\frac{\gamma_{i}^{\alpha_{1}+\beta_{1}}}{\mathrm{e}^{\frac{\bar{m}_{k i}\left(l_{1}+M\right) \gamma_{i}}{\bar{\Omega}_{k i}}}}\right\}-\left(k_{1}+k_{2}\right) \frac{\gamma_{i}^{\alpha_{1}-\left(k_{1}+k_{2} l_{1}\right) \beta_{1}}}{\mathrm{e}^{\frac{\bar{m}_{k i}\left(l_{1}+M\right) \gamma_{i}}{\bar{\Omega}_{k i}}}}\right],
$$

where $\beta_{1}=\left(v_{1}+v_{2}\right)(M-1)$ and $T_{m_{1}}=\left(a_{1} a_{2}\right)^{M-1} \sum_{v_{1}=0}^{(M-1)\left(\bar{m}_{1 k}-1\right)} \sum_{v_{2}=0}^{(M-1)\left(\bar{m}_{1 i}-1\right)} B_{1} D_{1}$.

Similarly, defining $b_{\min } \triangleq \min _{1 \leq l \leq L} \gamma_{\Sigma_{d_{l}}}$ for Cell-2, the PDF of $b_{\min }$ can be defined as

$$
\begin{aligned}
f_{b_{\min }}\left(\gamma_{l}\right) & =L f_{\gamma_{\Sigma_{d_{l}}}}\left(\gamma_{l}\right)\left[1-F_{\gamma_{\Sigma_{d_{l}}}}\left(\gamma_{l}\right)\right]^{L-1} \\
& =L f_{\gamma_{\Sigma_{d_{l}}}}\left(\gamma_{l}\right)\left[1-\int_{0}^{\gamma_{l}} f_{\gamma_{\Sigma_{d_{l}}}}\left(\gamma_{l}\right) \mathrm{d} \gamma_{l}\right]^{L-1}
\end{aligned}
$$

Substituting the values of $f_{\gamma_{\Sigma_{d l}}}\left(\gamma_{l}\right)$ in Equation (34) and using the identity of Equation (27), we have

$$
f_{b_{\min }}\left(\gamma_{l}\right)=L W Q_{m_{2}} R_{m_{2}} T_{m_{2}}\left[\frac{\bar{m}_{w l}}{\bar{\Omega}_{w l}}\left\{\frac{\gamma_{l}^{\alpha_{2}+\beta_{2}}}{\mathrm{e}^{\frac{\bar{m}_{w l}\left(\tau_{1}+L\right) \gamma_{l}}{\bar{\Omega}_{w l}}}}\right\}-\left(k_{1}+k_{2}\right) \frac{\gamma_{l}^{\alpha_{2}-\left(k_{1}+k_{2} l_{1}\right) \beta_{2}}}{\mathrm{e}^{\frac{\bar{m}_{w l}\left(\tau_{1}+L\right) \gamma_{l}}{\bar{\Omega}_{w l}}}}\right],
$$

where $\beta_{2}=\left(u_{1}+u_{2}\right)(L-1)$ and $T_{m_{2}}=\left(b_{1} b_{2}\right)^{L-1} \sum_{u_{1}=0}^{(L-1)\left(\bar{m}_{1 w}-1\right)} \sum_{u_{2}=0}^{(L-1)\left(\bar{m}_{1 /}-1\right)} B_{2} D_{2}$.

3) The PDF of SINR for Eavesdropper Channels: In the case of Cell-1, let $d_{\text {max }} \triangleq \max _{1 \leq j \leq N} \gamma_{\Sigma_{e_{j}}}$, then the PDF of $d_{\max }$ can be defined as [3] [24]

$$
f_{d_{\max }}\left(\gamma_{j}\right)=N f_{\gamma_{\Sigma_{e_{j}}}}\left(\gamma_{j}\right)\left[F_{\gamma_{\Sigma_{e_{j}}}}\left(\gamma_{i}\right)\right]^{N-1}=N f_{\gamma_{\Sigma_{e_{j}}}}\left(\gamma_{j}\right)\left[\int_{0}^{\gamma_{j}} f_{\gamma_{\Sigma_{e_{j}}}}\left(\gamma_{j}\right) \mathrm{d} \gamma_{j}\right]^{N-1}
$$

Substituting the values of $f_{\gamma_{\Sigma_{e_{j}}}}\left(\gamma_{j}\right)$ in Equation (36) and using the identity of Equation (27), we have

$$
f_{d_{\max }}\left(\gamma_{j}\right)=N K Q_{e_{1}} R_{e_{1}} T_{e_{1}}\left[\frac{\bar{m}_{k j}}{\bar{\Omega}_{k j}}\left\{\frac{\gamma_{j}^{\lambda_{1}+\beta_{3}}}{\mathrm{e}^{\frac{\bar{k}_{k j}\left(l_{1}+N\right) \gamma_{j}}{\bar{\Omega}_{k j}}}}\right\}-\left(k_{1}+k_{3}\right) \frac{\gamma_{j}^{\lambda_{1}-\left(k_{1}+k_{3} l_{1}\right) \beta_{3}}}{\mathrm{e}^{\frac{\bar{m}_{k j}\left(l_{1}+N\right) \gamma_{j}}{\bar{\Omega}_{k j}}}}\right],
$$

where $\beta_{3}=\left(v_{1}+v_{3}\right)(N-1)$ and $T_{e_{1}}=\left(a_{1} a_{3}\right)^{N-1} \sum_{v_{1}=0}^{(N-1)\left(\bar{m}_{1 k}-1\right)} \sum_{v_{3}=0}^{(N-1)\left(\bar{m}_{1 j}-1\right)} B_{1} G_{1}$.

Similarly, defining $b_{\max } \triangleq \max _{1 \leq q \leq Q} \gamma_{\Sigma_{e_{q}}}$ for Cell-2, the PDF of $b_{\max }$ can be defined as

$$
f_{b_{\max }}\left(\gamma_{q}\right)=Q f_{\gamma_{\Sigma_{e_{q}}}}\left(\gamma_{q}\right)\left[F_{\gamma_{\Sigma_{e_{q}}}}\left(\gamma_{q}\right)\right]^{Q-1}=Q f_{\gamma_{\Sigma_{e_{q}}}}\left(\gamma_{q}\right)\left[\int_{0}^{\gamma_{q}} f_{\gamma_{\Sigma_{e_{q}}}}\left(\gamma_{q}\right) \mathrm{d} \gamma_{q}\right]^{Q-1}
$$

Substituting the values of $f_{\gamma_{\Sigma_{e_{q}}}}\left(\gamma_{q}\right)$ in Equation (38) and using the identity of Equation (27), we have

$$
f_{b_{\max }}\left(\gamma_{q}\right)=Q W Q_{e_{2}} R_{e_{2}} T_{e_{2}}\left[\frac{\bar{m}_{w q}}{\bar{\Omega}_{w q}}\left\{\frac{\gamma_{q}^{\lambda_{2}+\beta_{4}}}{\mathrm{e}^{\frac{\bar{m}_{w q}\left(\tau_{1}+Q\right) \gamma_{q}}{\bar{\Omega}_{w q}}}}\right\}-\left(t_{1}+t_{3}\right) \frac{\gamma_{q}^{\lambda_{2}-\left(t_{1}+t_{3} \tau_{1}\right) \beta_{4}}}{\mathrm{e}^{\frac{\bar{m}_{w q}\left(\tau_{1}+Q\right) \gamma_{q}}{\bar{\Omega}_{w q}}}}\right],
$$


where $\beta_{4}=\left(d_{1}+d_{3}\right)(Q-1)$ and $T_{e_{2}}=\left(b_{1} b_{3}\right)^{Q-1} \sum_{d_{1}=0}^{(Q-1)\left(\bar{m}_{1 w}-1\right)} \sum_{d_{3}=0}^{(Q-1)\left(\bar{m}_{1 q}-1\right)} B_{1} G_{2}$.

\section{Probability of Non-Zero Secrecy Multicast Capacity}

The probability of non-zero secrecy multicast capacity for Cell-1 can be defined as

$$
\operatorname{Pr}\left(C_{\text {smcast }}>0\right)_{\text {Cell- } 1}=\int_{0}^{\infty} f_{d_{\min }}\left(\gamma_{i}\right) \int_{0}^{\gamma_{i}} f_{d_{\max }}\left(\gamma_{j}\right) \mathrm{d} \gamma_{j} \mathrm{~d} \gamma_{i}
$$

Substituting the values of $f_{d_{\min }}\left(\gamma_{i}\right)$ and $f_{d_{\max }}\left(\gamma_{j}\right)$ in Equation (40) and performing integration, the closed-form analytical expression for the probability of non-zero secrecy multicast capacity is given by

$$
\begin{aligned}
\operatorname{Pr}\left(C_{\text {smcast }}>0\right)_{\text {Cell- } 1}= & K^{2} Q_{m_{1}} R_{m_{1}} T_{m_{1}} Q_{e_{1}} R_{e_{1}} T_{e_{1}} M N\left[\frac{\bar{m}_{k i} \Lambda_{1} n_{3} !}{\bar{\Omega}_{k i} \mu_{2}^{\left(n_{3}+1\right)}}-\frac{k_{12} \Lambda_{1} n_{4} !}{\mu_{2}^{\left(n_{4}+1\right)}}\right. \\
& \left.-\frac{\bar{m}_{k i} \theta_{1} \varphi_{1} !}{\left.\bar{\Omega}_{k i} \mu_{12} \mu_{1}+1\right)}+\frac{k_{12} \theta_{1} \varphi_{3} !}{\mu_{12}^{\left(\varphi_{3}+1\right)}}+\frac{\bar{m}_{k j} \theta_{2} \varphi_{2} !}{\bar{\Omega}_{k j} \mu_{12}^{\left(\varphi_{2}+1\right)}}-\frac{k_{12} \theta_{2} \varphi_{4} !}{\mu_{12}^{\left(\varphi_{2}+1\right)}}\right],
\end{aligned}
$$

where $\theta_{1}=\sum_{q_{1}=0}^{n_{1}} \frac{\bar{m}_{k i} n_{1} !}{\Omega_{k i} q_{1} ! \mu_{1}^{n_{1}-q_{1}+1}}, \quad \theta_{2}=\sum_{q_{2}=0}^{n_{2}} \frac{k_{12} ! k_{12}}{q_{2} ! \mu_{1}^{k_{12}-q_{2}+1}}$,

$$
\begin{aligned}
& \Lambda_{1}=\frac{\bar{m}_{k i} n_{1}}{\bar{\Omega}_{k i} \mu_{1}^{\left(n_{1}+1\right)}}-\frac{k_{12}^{2}}{\mu_{1}^{\left(n_{2}+1\right)}}, \mu_{12}=\mu_{1}+\mu_{2}, \quad k_{12}=k_{1}+k_{2}, \quad \varphi_{1}=q_{1}+n_{3}, \\
& \varphi_{2}=q_{2}+n_{3}, \varphi_{3}=q_{1}+n_{4}, \quad \varphi_{4}=q_{2}+n_{4}, \quad \bar{\mu}=K N-K+l_{1}, \quad \mu_{1}=\frac{m^{k j}}{\bar{\Omega}_{k j}}(\bar{\mu}+1), \\
& \mu_{2}=\frac{m^{k i}}{\bar{\Omega}_{k i}}\left(M+l_{1}\right), \quad n_{1}=k_{12}(\bar{\mu}+1), \quad n_{3}=\alpha_{1}+\beta_{1} \text { and } n_{4}=\left(\alpha_{1}-l_{1} k_{12}\right) \beta_{2} .
\end{aligned}
$$

Similarly, the probability of non-zero secrecy multicast capacity for Cell-2 can be defined as

$$
\operatorname{Pr}\left(C_{\text {smcast }}>0\right)_{\text {Cell- }-2}=\int_{0}^{\infty} f_{b_{\min }}\left(\gamma_{l}\right) \int_{0}^{\gamma_{l}} f_{b_{\max }}\left(\gamma_{q}\right) \mathrm{d} \gamma_{q} \mathrm{~d} \gamma_{l}
$$

Substituting the values of $f_{b_{\min }}\left(\gamma_{l}\right)$ and $f_{b_{\max }}\left(\gamma_{q}\right)$ in Equation (42) and performing integration, the closed-form analytical expression for the probability of non-zero secrecy multicast capacity is given by

$$
\begin{aligned}
\operatorname{Pr}\left(C_{\text {smcast }}>0\right)_{\text {Cell- } 2}= & W^{2} Q_{m_{2}} R_{m_{2}} T_{m_{2}} Q_{e_{2}} R_{e_{2}} T_{e_{2}} L Q\left[\frac{\bar{m}_{w l} G_{2} \eta_{3} !}{\bar{\Omega}_{w l} v_{2}^{\left(\eta_{3}+1\right)}}-\frac{t_{12} G_{2} \eta_{4} !}{v_{2}^{\left(\eta_{4}+1\right)}}\right. \\
& \left.-\frac{\bar{m}_{w l} \vartheta_{1} \varphi_{1} !}{\bar{\Omega}_{w l} l_{12}^{\left(\varphi_{1}+1\right)}}+\frac{t_{12} \vartheta_{1} \varphi_{3} !}{v_{12}^{\left(\varphi_{3}+1\right)}}+\frac{\bar{m}_{w q} \vartheta_{2} \varphi_{2} !}{\bar{\Omega}_{w q} v_{12}^{\left(\varphi_{2}+1\right)}}-\frac{t_{12} \vartheta_{2} \varphi_{4} !}{v_{12}^{\left(\varphi_{4}+1\right)}}\right],
\end{aligned}
$$

where $v_{12}=v_{1}+v_{2}, \vartheta_{1}=\sum_{\sigma_{1}=0}^{\eta_{1}} \frac{\bar{m}_{w l} \eta_{1} !}{\Omega_{w l} \sigma_{1} ! v_{1}^{\eta_{1}-\sigma_{1}+1}}, \vartheta_{2}=\sum_{\sigma_{2}=0}^{t_{12}} \frac{t_{12} ! t_{12}}{\sigma_{2} ! v_{1}^{t_{12}-\sigma_{2}+1}}$,

$$
\begin{aligned}
& \Lambda_{2}=\frac{\bar{m}_{w l} n_{1}}{\bar{\Omega}_{w l} v_{1}^{\left(\eta_{1}+1\right)}}-\frac{t_{12}^{2}}{v_{1}^{\left(\eta_{2}+1\right)}}, \quad t_{12}=t_{1}+t_{2}, \quad \varphi_{1}=q_{1}+\eta_{3}, \quad \varphi_{2}=q_{2}+\eta_{3}, \quad \varphi_{3}=q_{1}+\eta_{4}, \\
& \varphi_{4}=q_{2}+\eta_{4}, \quad \bar{v}=W Q-W+\tau_{1}, \quad v_{1}=\frac{m^{w q}}{\bar{\Omega}_{w q}}(\bar{v}+1), \quad v_{2}=\frac{m^{w l}}{\bar{\Omega}_{w l}}\left(L+\tau_{1}\right), \\
& \eta_{3}=\lambda_{1}+\beta_{3} \text { and } \eta_{4}=\left(\lambda_{1}-\tau_{1} t_{12}\right) \beta_{4} .
\end{aligned}
$$




\section{Secure Outage Probability for Multicasting}

In the case of Cell-1, the secure outage probability for multicasting can be defined as

$$
P_{\text {out }}\left(R_{\text {smcast }}\right)_{\text {Cell- } 1}=1-\int_{0}^{\infty} f_{d_{\max }}\left(\gamma_{j}\right) \times\left\{\int_{x_{1}}^{\infty} f_{d_{\min }}\left(\gamma_{i}\right) \mathrm{d} \gamma_{i}\right\} \mathrm{d} \gamma_{j},
$$

where $x=\mathrm{e}^{2 R_{\text {smcast }}\left(1+\gamma_{j}\right)-1}$ and $R_{\text {smcast }}$ denotes the target secrecy multicast rate. Substituting the values of $f_{d_{\min }}\left(\gamma_{i}\right)$ and $f_{d_{\max }}\left(\gamma_{j}\right)$ in Equation (44) and performing the integration the closed-form analytical expression for $P_{\text {out }}\left(R_{s}\right)_{\text {Cell-1 }}$ is given by

$$
\begin{aligned}
& P_{\text {out }}\left(R_{\text {smcast }}\right)_{\text {Cell- } 1}=K^{2} Q_{m_{1}} R_{m_{1}} T_{m_{1}} Q_{e_{1}} R_{e_{1}} T_{e_{1}} \phi_{m_{1}} \frac{\bar{m}_{k i} \bar{m}_{k j} k_{12}}{\bar{\Omega}_{k i} \bar{\Omega}_{k j}}\left[\frac{\phi_{e_{1}} k_{12} r_{m_{1}} !}{\mu_{m_{t}}^{r_{m_{3}}+1}}\right. \\
& \left.+\frac{\phi_{e_{1}} r_{m_{1}} ! r_{m_{2}} !}{\mu_{m_{t}}^{r_{m_{1}}+r_{m_{2}}+2}}-\frac{\phi_{m_{1}} r_{m_{3}} !}{\mu_{m_{t}}^{r_{m_{3}}+1}}\right]
\end{aligned}
$$

where $\mu_{m_{t}}=\mu_{1}+\mu_{2} \mathrm{e}^{2 R_{\text {smcast }}}, \quad \phi_{m_{1}}=\sum_{\eta_{1}=0}^{n_{3}} \sum_{s_{1}=0}^{\eta_{1}}\left(\begin{array}{l}r_{1} \\ s_{1}\end{array}\right) \frac{R_{t}^{r_{1}-s_{1}} n_{3} ! \mathrm{e}^{2 \eta_{1} R_{\text {smeast }}}}{r_{1} ! \mu_{2}^{n_{3}-r_{1}+1}}$ and $\phi_{e_{1}}=\sum_{r_{2}=0}^{n_{4}} \sum_{s_{2}=0}^{r_{2}}\left(\begin{array}{c}r_{2} \\ s_{2}\end{array}\right) \frac{R_{t}^{r_{2}-s_{2}} n_{4} ! \mathrm{e}^{2 r_{2} R_{s}}}{r_{2} ! \mu_{2}^{n_{4}-r_{2}+1}}, r_{m_{1}}=r_{1}+n_{1}, \quad r_{m_{2}}=r_{2}+n_{1}, \quad r_{m_{3}}=r_{1}+n_{2}$, $r_{m_{4}}=r_{2}+n_{2}$ and $R_{t}=-1+2^{2 R_{\text {smcast }}}$.

Similarly, in the case of Cell-2, the secure outage probability for multicasting can be defined as

$$
P_{\text {out }}\left(R_{\text {smcast }}\right)_{\text {Cell- } 2}=1-\int_{0}^{\infty} f_{b_{\max }}\left(\gamma_{q}\right) \times\left\{\int_{x_{1}}^{\infty} f_{b_{\min }}\left(\gamma_{l}\right) \mathrm{d} \gamma_{l}\right\} \mathrm{d} \gamma_{q},
$$

Substituting the values of $f_{b_{\min }}\left(\gamma_{l}\right)$ and $f_{b_{\max }}\left(\gamma_{q}\right)$ in Equation (46) and performing integration, the closed-form analytical expression for the secure outage probability for multicasting is given by

$$
\begin{aligned}
P_{\text {out }}\left(R_{\text {smcast }}\right)_{\text {Cell-2 }}= & Q^{2} Q_{m_{2}} R_{m_{2}} T_{m_{2}} Q_{e_{2}} R_{e_{2}} T_{e_{2}} \phi_{m_{2}} \frac{\bar{m}_{w l} \bar{m}_{w q} t_{12}}{\bar{\Omega}_{w l} \bar{\Omega}_{w q}}\left[\frac{\phi_{e_{2}} t_{12} r_{m_{2}} !}{\mu_{e_{t}}^{r_{e_{3}}+1}}\right. \\
& \left.+\frac{\phi_{e_{2}} r_{e_{1}} ! r_{e_{2}} !}{\mu_{e_{t}}^{r_{e_{1}}+r_{e_{2}}+2}}-\frac{\phi_{m_{2}} r_{e_{2}} !}{\mu_{e_{t}}^{r_{e_{2}}+1}}\right],
\end{aligned}
$$

where $\mu_{e_{t}}=v_{1}+v_{2} \mathrm{e}^{2 R_{\text {smast }}}, \quad \phi_{m_{2}}=\sum_{\varepsilon_{1}=0}^{\eta_{3}} \sum_{\varepsilon_{1}=0}^{\varepsilon_{1}}\left(\begin{array}{l}\varepsilon_{1} \\ \varepsilon_{1}\end{array}\right) \frac{R_{t}^{\varepsilon_{1}-\varepsilon_{1}} \eta_{3} ! \mathrm{e}^{2 \varepsilon_{1} R_{\text {smcast }}}}{\varepsilon_{1} ! v_{2}^{\eta_{3}-\varepsilon_{1}+1}}$ and $\phi_{e_{2}}=\sum_{\varepsilon_{2}=0}^{\eta_{4}} \sum_{\varepsilon_{2}=0}^{\varepsilon_{2}}\left(\begin{array}{c}\varepsilon_{2} \\ \varepsilon_{2}\end{array}\right) \frac{R_{t}^{\varepsilon_{2}-\varepsilon_{2}} \eta_{4} ! \mathrm{e}^{2 \varepsilon_{2} R_{\text {smeast }}}}{\varepsilon_{2} ! v_{2}^{\eta_{4}-\varepsilon_{2}+1}}, r_{e_{1}}=\varepsilon_{1}+\eta_{1}, \quad r_{e_{2}}=\varepsilon_{2}+\eta_{1}$, $r_{e_{3}}=\varepsilon_{1}+\eta_{2}$ and $r_{e_{4}}=\varepsilon_{2}+\eta_{2}$.

\section{Numerical Results}

In the case of Cell-1, Figure 2 shows the probability of non-zero secrecy multicast capacity, $\operatorname{Pr}\left(C_{\text {smcast }}>0\right)$, as a function of the average SINR of the multicast channel for selected values of $M$ and $N$. This figure describes the effects 


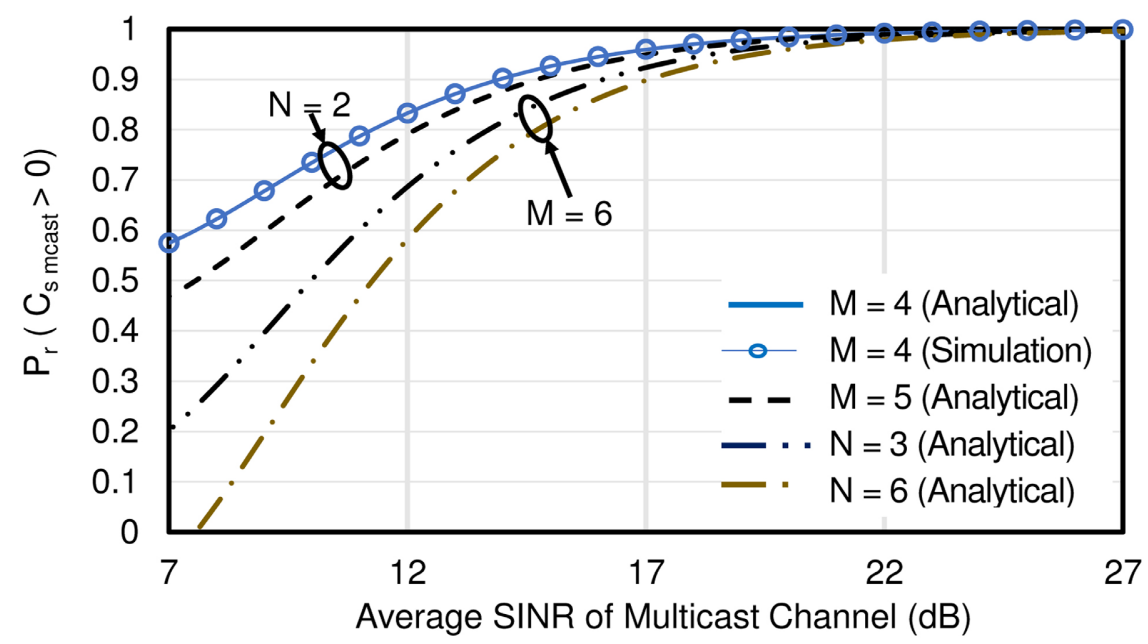

Figure 2. Effect of the number of multicast user, $M$, and eavesdropper, $N$, for Cell-1 on the $\operatorname{Pr}\left(C_{\text {smcast }}>0\right)$ for $N=2$ and $M=5$, respectively, with $K=4, n_{r_{1}}=n_{e_{1}}=6$, $m=1.5, \rho_{m_{1}}=\rho_{e_{1}}=0.5, I_{m_{1}}=1$ watt, $I_{e_{1}}=3$ watt and $w_{e}=-5 \mathrm{~dB}$.

of $M$ and $N$ on the $\operatorname{Pr}\left(C_{\text {smcast }}>0\right)$ for selected values of system parameters. We see that the $\operatorname{Pr}\left(C_{\text {smcast }}>0\right)$ decreases, if the values of $M$ increases from 4 (indicated by the solid line) to 5 (indicated by the dash line) keeping the value of $N$ equal to 2. Similarly, the $\operatorname{Pr}\left(C_{\text {smcast }}>0\right)$ also decreases, if the values of $N$ increases from 3 (indicated by the long dash dot-dot line) to 6 (indicated by the long dash dot line) keeping the value of $M$ equal to 6 . Hence, it can be concluded that the $\operatorname{Pr}\left(C_{\text {smcast }}>0\right)$ decreases with $M$ and $N$. This is because, the increasing in $M$, the bandwidth of each user decreases which causes decreasing in the secrecy multicast capacity. Moreover, the increasing in $N$, the probability of eavesdropping increases which causes the decreasing in $\operatorname{Pr}\left(C_{\text {smcast }}>0\right)$. Finally, matching between the simulation and analytical results justifies the validity of derived expression.

The $\operatorname{Pr}\left(C_{\text {smcast }}>0\right)$ is shown in Figure 3 as a function of the average SINR of the multicast channel for selected values of $m$ and $K$. This figure describes the effects of $m$ and $K$ on the $\operatorname{Pr}\left(C_{\text {smcast }}>0\right)$ for selected values of system parameters. We see that the $\operatorname{Pr}\left(C_{\text {smcast }}>0\right)$ decreases if the values of $m$ increases from 1.8 (indicated by the dash line) to 2.5 (indicated by the solid line) keeping the value of $K$ equal to 2. $\operatorname{Pr}\left(C_{\text {smcast }}>0\right)$ increases if the values of $K$ increases from 4 (indicated by the long dash dot line) to 6 (indicated by the long dash dot-dot line) keeping the value of $m$ equal to 1.8 . Hence, it can be concluded that the $\operatorname{Pr}\left(C_{\text {smcast }}>0\right)$ increases with $K$ and but decreases with $m$. This is because, the increasing in $K$ provides additional diversity which enhances the secrecy multicast capacity and the increasing in $m$ reduces the SINR which degrades the secrecy multicast capacity.

Figure 4 shows $\operatorname{Pr}\left(C_{\text {smcast }}>0\right)$ as a function of the average SINR of the multicast channel for selected values of $\rho_{m_{1}}, n_{\eta_{1}}$ and $n_{e_{1}}$. This figure describes the effects of $\rho_{m_{1}}, n_{\eta_{1}}$ and $n_{e_{1}}$ on the $\operatorname{Pr}\left(C_{\text {smcast }}>0\right)$ for selected values of 


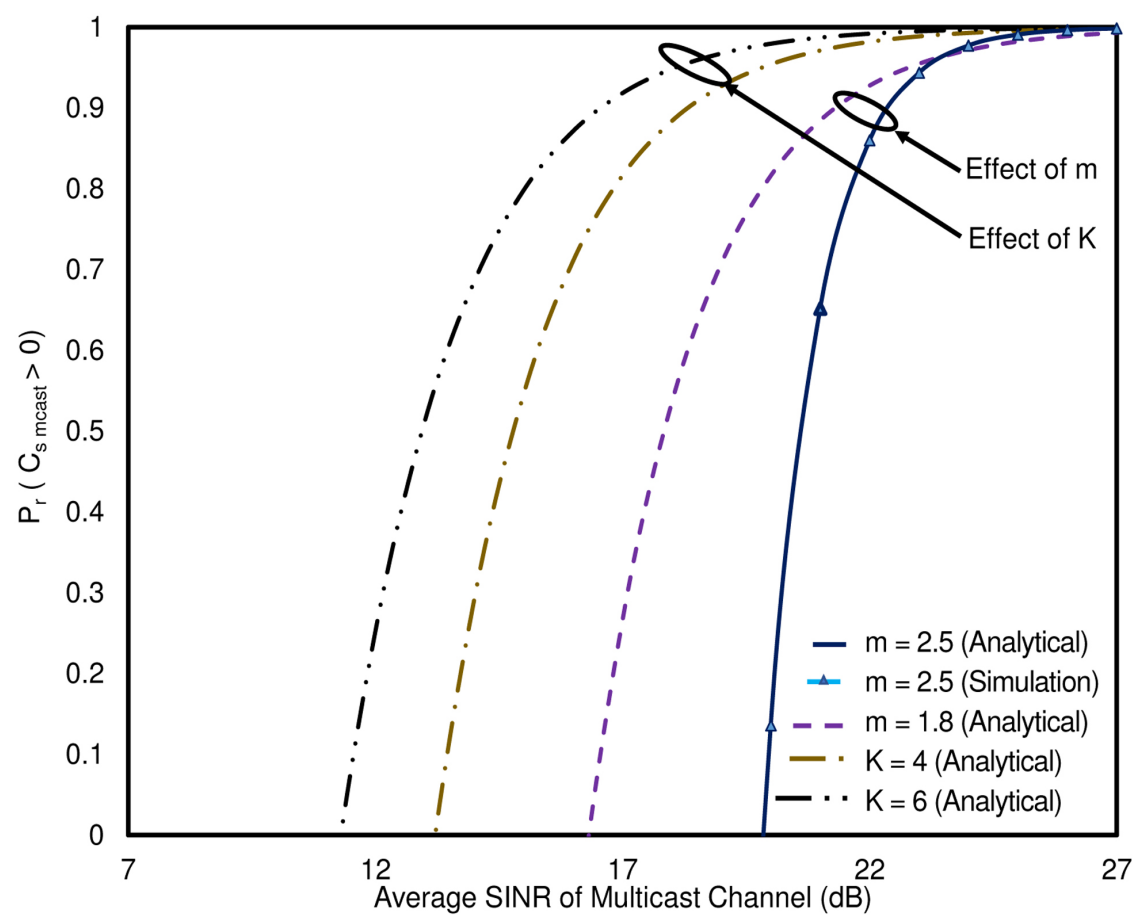

Figure 3. Effect of the fading parameter, $m$, and the number of relays, $K$, for Cell-1 on the $\operatorname{Pr}\left(C_{\text {smcast }}>0\right)$ for $K=2$ and $m=1.8$, respectively, with $M=5, N=4$, $n_{r_{1}}=n_{e_{1}}=6, \rho_{m_{1}}=\rho_{e_{1}}=0.5, I_{m_{1}}=1$ watt, $I_{e_{1}}=3$ watt and $w_{e}=-5 \mathrm{~dB}$.

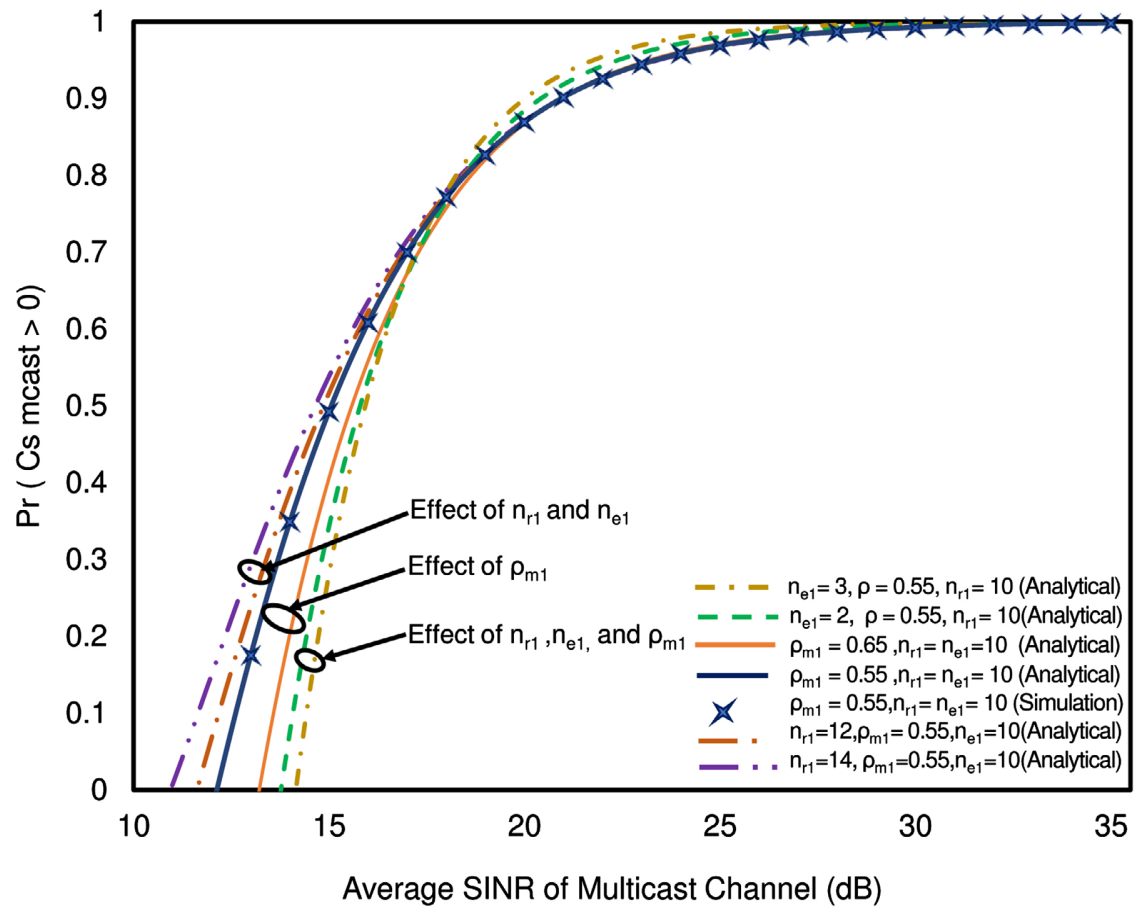

Figure 4. Effect of the correlation coefficient, $\rho_{m_{1}}$, and the number of receiving antennas at the multicast users, $n_{\eta_{1}}$, and the eavesdropper, $n_{e_{1}}$, for Cell-1 on the $\operatorname{Pr}\left(C_{\text {smcast }}>0\right)$ for $n_{r_{1}}=n_{e_{1}}=10$ and $\rho_{m_{1}}=0.55$, respectively, with $M=5, N=4, K=2, m=1.5$, $I_{m_{1}}=1$ watt, $I_{e_{1}}=1$ watt and $\omega_{e}=-5 \mathrm{~dB}$. 
system parameters. It is observed that the $\operatorname{Pr}\left(C_{\text {smcast }}>0\right)$ decreases if the values of $\rho_{m_{1}}$ increases from 0.55 (indicated by the solid with asterisk line) to 0.65 (indicated by the solid line) with $n_{\eta_{1}}=n_{e_{1}}=10$. The $\operatorname{Pr}\left(C_{\text {smcast }}>0\right)$ increases if the values of $n_{\eta}$ increases from 12 (indicated by the long dash dot line) to 14 (indicated by the long dash dot-dot line) with $\rho=0.55$. The $\operatorname{Pr}\left(C_{\text {smcast }}>0\right)$ decreases if the values of $n_{e_{1}}$ increases from 2 (indicated by the dash line) to 3 (indicated by the dash-dot line) with $\rho_{m_{1}}=0.55$ and $n_{r_{1}}=10$. In conclusion, $\operatorname{Pr}\left(C_{\text {smcast }}>0\right)$ increases with $n_{r_{1}}$ and decreases with $\rho_{m_{1}}$ and $n_{e_{1}}$ as one expects.

The $\operatorname{Pr}\left(C_{\text {smcast }}>0\right)$ is shown in Figure 5 as a function of the average SINR of the multicast channel for selected values of $\omega_{e}, I_{m_{1}}$, and $I_{e_{1}}$. This figure illustrates the effects of $\omega_{e}, I_{m_{1}}$, and $I_{e_{1}}$ on the $\operatorname{Pr}\left(C_{\text {smcast }}>0\right)$ for selected values of system parameters. It is observed that the $\operatorname{Pr}\left(C_{\text {smcast }}>0\right)$ increases, if the values of $I_{e_{1}}$ increases from 2 watt (indicated by the long dash dot line) to 3 watt (indicated by the long dash dot-dot line) keeping the value of $I_{m_{1}}$ and $\omega_{e}$ equal to 1 watt and $-5 \mathrm{~dB}$, respectively. Similarly, the $\operatorname{Pr}\left(C_{\text {smcast }}>0\right)$ also decreases, if the values of $I_{m_{1}}$ increases from 4 watt (indicated by the dash dot line) to 6 watt (indicated by the dash line) keeping the value of $\omega_{e}$ and $I_{e_{1}}$ equal to $-5 \mathrm{~dB}$ and 1 watt, respectively. Moreover, the $\operatorname{Pr}\left(C_{\text {smcast }}>0\right)$ decreases, if the values of $\omega_{e}$ increases from $-10 \mathrm{~dB}$ (indicated by the solid line) to -7 $\mathrm{dB}$ (indicated by the solid line with asterisk) keeping the value of $I_{m_{1}}$ and $I_{e_{1}}$ equal to 1 watt and 3 watt, respectively. Hence, it can be concluded that the

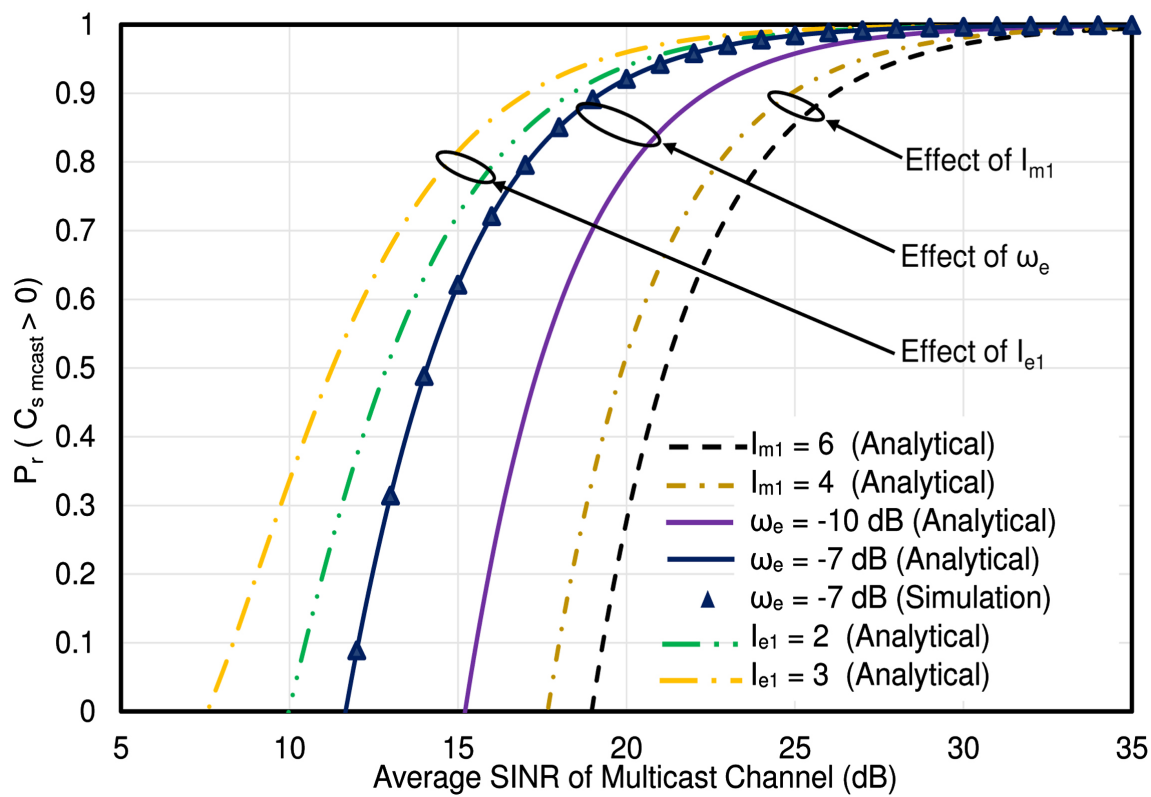

Figure 5. Effect of the SINR of evesdropper channel, $\omega_{e}$, and the effect of interference at the multicast users, $I_{m_{1}}$, and the effect of interference at the eavesdropper, $I_{e_{1}}$, created from Cell-2 on the $\operatorname{Pr}\left(C_{\text {smcast }}>0\right)$ for $I_{m_{1}}=1$ watt and $I_{e_{1}}=3$ watt, $\omega_{e}=-5 \mathrm{~dB}$ and $I_{e_{1}}=3$ watt, and $\omega_{e_{1}}=-5 \mathrm{~dB}$ and $I_{m_{1}}=1$ watt, respectively, with $M=5, N=4$, $\rho_{m_{1}}=0.5, K=2, n_{\eta_{1}}=n_{e_{1}}=10$ and $m=1.5$. 
$\operatorname{Pr}\left(C_{\text {smcast }}>0\right)$ increases with $I_{e_{1}}$ and decreases with $\omega_{e}$ and $I_{m_{1}}$. This is because, the interference at the eavesdropper channel and the degradation of SINR in the eavesdropper channel degrades the capacity of eavesdropper channel which causes the improvement in the secrecy capacity for multicasting and interference at the multicast channel also degrades the capacity of multicast channels which degrades the secrecy capacity for multicasting.

Figure 6 shows the secure outage probability for multicasting, $P_{\text {out }}\left(R_{\text {smcast }}\right)$, as a function of the average SINR of the multicast channel for selected values of $M$ and $N$. This figure describes the effects of $M$ and $N$ on the $P_{\text {out }}\left(R_{\text {smcast }}\right)$ for selected values of system parameters. We see that the $P_{\text {out }}\left(R_{\text {smcast }}\right)$ increases, if the values of $M$ increases from 4 (indicated by the solid line) to 8 (indicated by the dash line) keeping the value of $N$ equal to 2 . Similarly, the $P_{\text {out }}\left(R_{\text {smcast }}\right)$ also increases, if the values of $N$ increases from 3 (indicated by the long dash dot-dot line) to 4 (indicated by the long dash dot line) keeping the value of $M$ equal to 6 . Hence, it can be concluded that the $P_{\text {out }}\left(R_{\text {smcast }}\right)$ increases with $M$ and $N$. This is because, the increasing in $M$, the bandwidth of each user decreases and the increasing in $N$, the probability of eavesdropping increases which increases the probability of outage.

The $P_{\text {out }}\left(R_{\text {smcast }}\right)$ is shown in Figure 7 as a function of the average SINR of the multicast channel for selected values of $m$ and $K$. This figure describes the effects of $m$ and $K$ on the $P_{\text {out }}\left(R_{\text {smcast }}\right)$ for selected values of system parameters. We see that the $P_{\text {out }}\left(R_{\text {smcast }}\right)$ increases if the values of $m$ increases from 1.8 (indicated by the solid line) to 2.5 (indicated by the dash line) keeping the value of $K$ equal to 2. $P_{\text {out }}\left(R_{\text {smcast }}\right)$ decreases if the values of $K$ increases from 4 (indicated by the long dash dot-dot line) to 6 (indicated by the long dash dot line) keeping the value of $m$ equal to 1.8 . Hence, it can be concluded that the

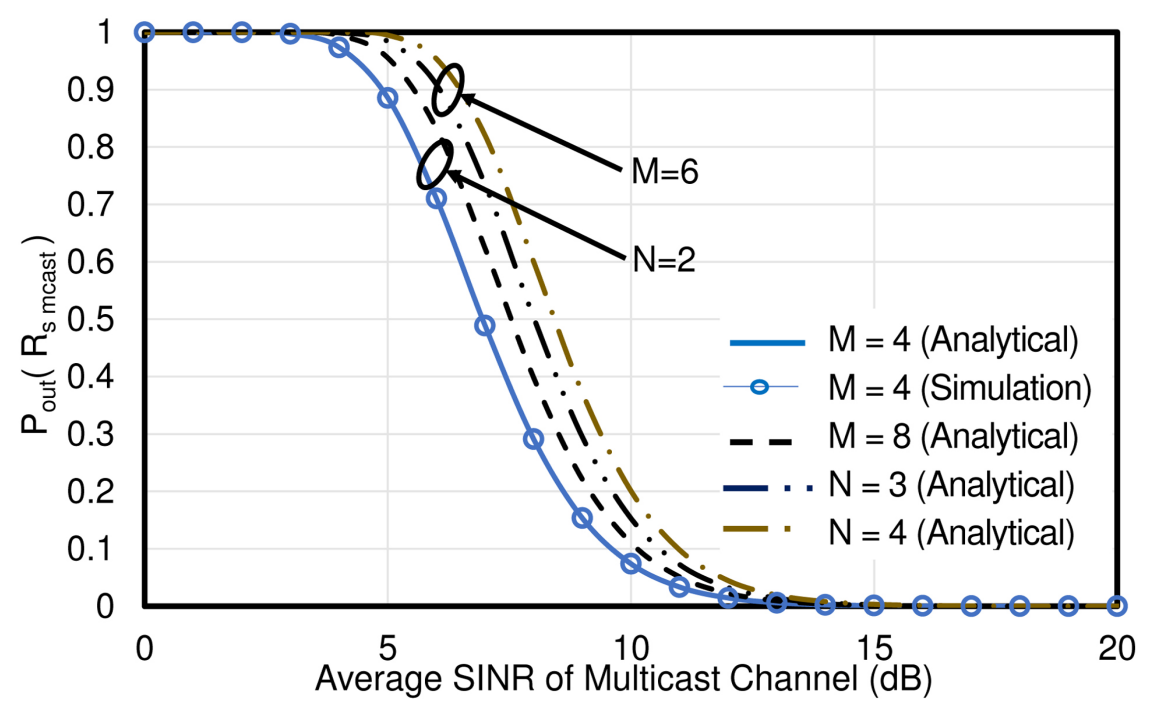

Figure 6. Effect of the number of multicast user, $M$, and eavesdropper, $N$, for Cell-1 on the $P_{\text {out }}\left(R_{\text {smcast }}\right)$ for $N=2$ and $M=6$, respectively, with $K=2, \quad n_{r_{1}}=n_{e_{1}}=2$, $m=1, \quad \rho_{m_{1}}=\rho_{e_{1}}=0.5, I_{m_{1}}=1$ watt, $I_{e_{1}}=3$ watt and $w_{e}=-5 \mathrm{~dB}$. 


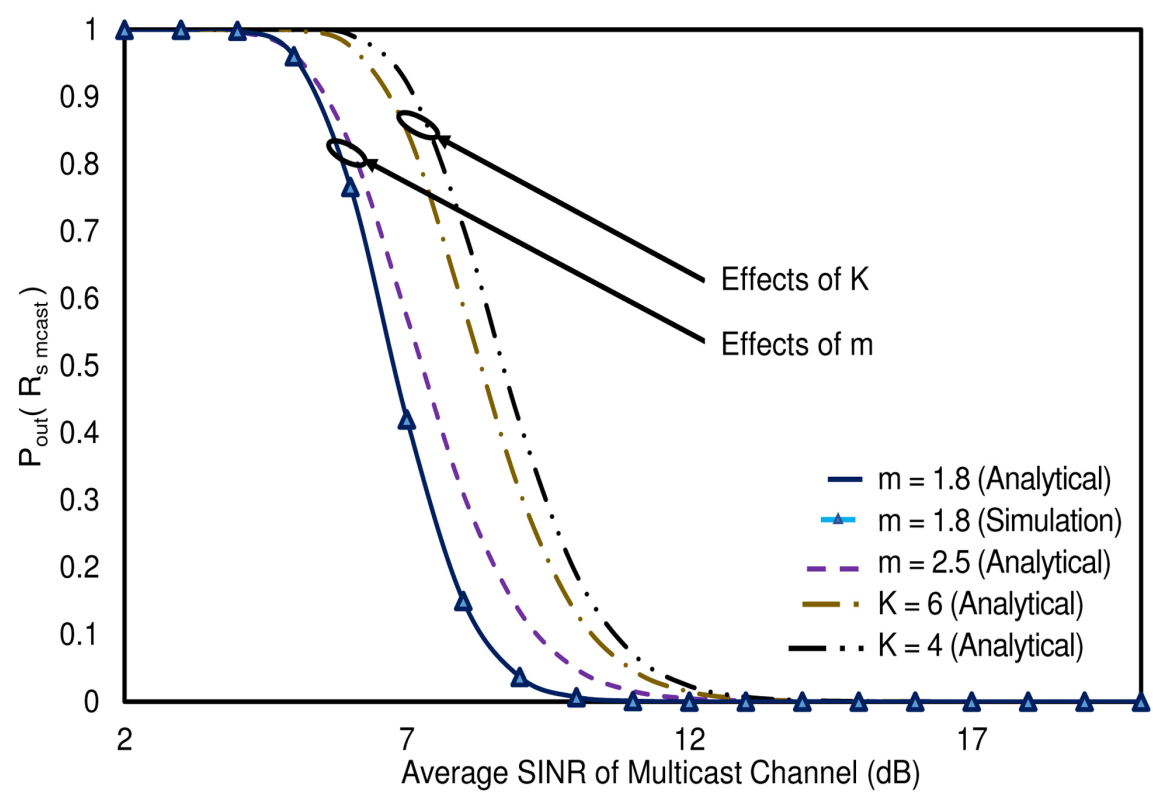

Figure 7. Effect of the fading parameter, $m$, and the number of relays, $K$, for Cell- 1 on the $P_{\text {out }}\left(R_{\text {smcast }}\right)$ for $K=2$ and $m=1.8$, respectively, with $M=5, N=2, n_{r_{1}}=n_{e_{1}}=2$, $\rho_{m_{1}}=\rho_{e_{1}}=0.5, I_{m_{1}}=1$ watt, $I_{e_{1}}=3$ watt and $w_{e}=-5 \mathrm{~dB}$.

$P_{\text {out }}\left(R_{\text {smcast }}\right)$ decreases with $K$ and increases with $m$ as one expects.

Figure 8 shows $P_{\text {out }}\left(R_{\text {smcast }}\right)$ as a function of the average SINR of the multicast channel for selected values of $\rho_{m_{1}}, n_{\eta_{1}}$ and $n_{e_{1}}$. This figure describes the effects of $\rho_{m_{1}}, n_{\eta_{1}}$ and $n_{e_{1}}$ on the $P_{\text {out }}\left(R_{\text {smcast }}\right)$ for selected values of system parameters. It is observed that the $P_{\text {out }}\left(R_{\text {smcast }}\right)$ increases if the values of $\rho_{m_{1}}$ increases from 0.50 (indicated by the solid with square line) to 0.60 (indicated by the solid line) with $n_{r_{1}}=n_{e_{1}}=10$. The $P_{\text {out }}\left(R_{\text {smcast }}\right)$ decreases if the values of $n_{r_{1}}$ increases from 2 (indicated by the long dash dot line) to 3 (indicated by the long dash dot-dot line) with $\rho=0.50$. The $P_{\text {out }}\left(R_{\text {smcast }}\right)$ increases if the values of $n_{e_{1}}$ increases from 2 (indicated by the dash line) to 3 (indicated by the dash-dot line) with $\rho_{m_{1}}=0.50$ and $n_{\eta_{1}}=2$. In conclusion, $P_{\text {out }}\left(R_{\text {smcast }}\right)$ decreases with $n_{\eta_{1}}$ and increases with $\rho_{m_{1}}$ and $n_{e_{1}}$.

The $P_{\text {out }}\left(R_{\text {smcast }}\right)$ is shown in Figure 9 as a function of the average SINR of the multicast channel for selected values of $I_{m_{2}}$, and $I_{e_{2}}$. This figure illustrates the effects of $I_{m_{2}}$, and $I_{e_{2}}$ on the $P_{\text {out }}\left(R_{\text {smcast }}\right)$. It is observed that the $P_{\text {out }}\left(R_{\text {smcast }}\right)$ decreases, if the values of $I_{e_{1}}$ increases from 3 watt (indicated by the long dash dot line) to 6 watt (indicated by the long dash dot-dot line) keeping the value of $I_{m_{1}}=2$ watt and $\omega_{e}=-5 \mathrm{~dB}$, respectively. Similarly, the $P_{\text {out }}\left(R_{\text {smcast }}\right)$ also increases, if the values of $I_{m_{1}}$ increases from 4 watt (indicated by the dash line) to 6 watt (indicated by the dash dot line) keeping the value of $\omega_{e}$ and $I_{e_{1}}$ equal to $-5 \mathrm{~dB}$ and 1 watt, respectively. Moreover, the $P_{\text {out }}\left(R_{\text {smcast }}\right)$ increases, if the values of $\omega_{e}$ increases from $-10 \mathrm{~dB}$ (indicated by the solid line) to $-7 \mathrm{~dB}$ (indicated by the solid line with asterisk) keeping the value of $I_{m_{1}}$ and $I_{e_{1}}$ equal to 2 watt and 1 watt, respectively. Hence, it can be 


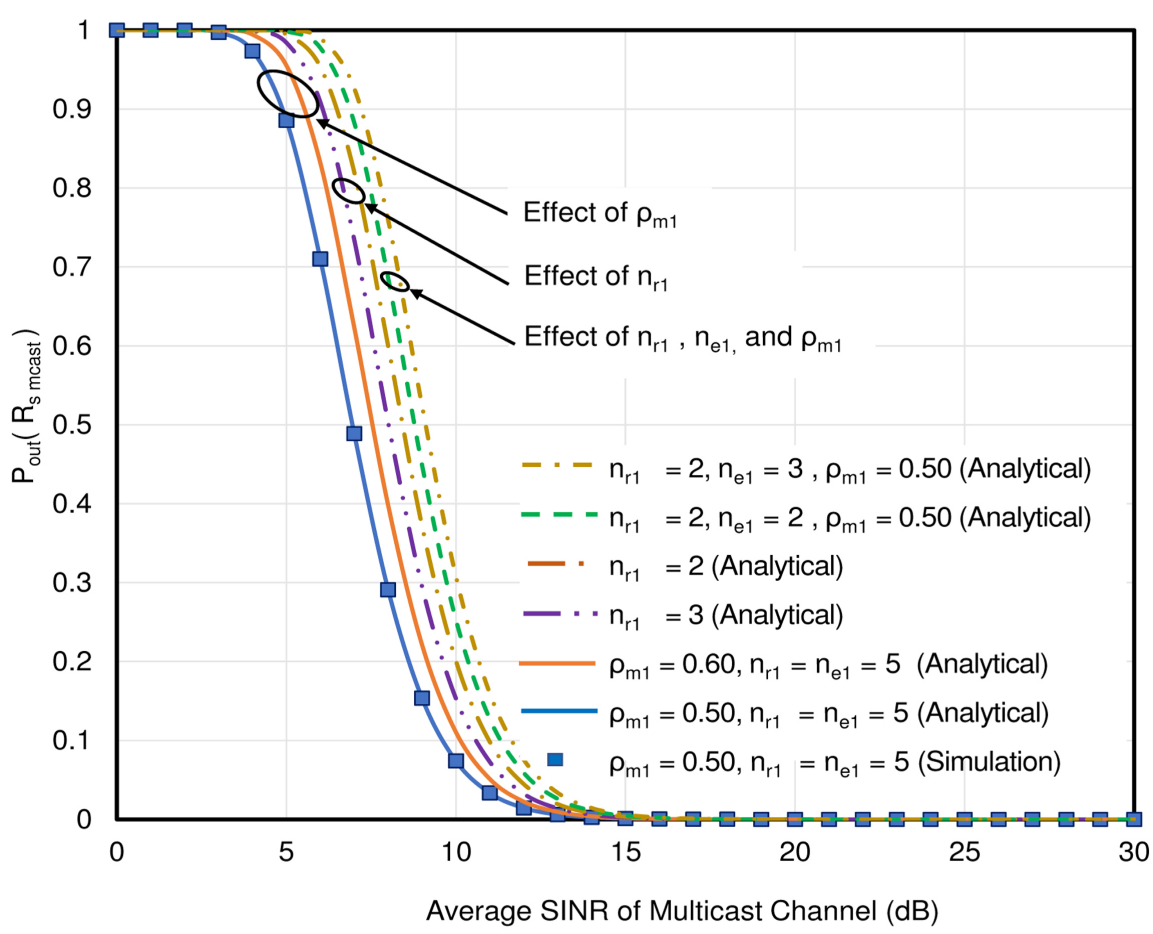

Figure 8. Effect of the correlation coefficient, $\rho_{m_{1}}$, and the number of receiving antennas at the multicast users, $n_{r_{1}}$, and the eavesdropper, $n_{e_{1}}$, for Cell-1 on the $P_{\text {out }}\left(R_{\text {smcast }}\right)$ for $n_{r_{1}}=n_{e_{1}}=2$ and $\rho_{m_{1}}=0.55$, respectively, with $M=5, N=4, K=2, m=1.5$, $I_{m_{1}}=1$ watt, $I_{e_{1}}=1$ watt and $\omega_{e}=-5 \mathrm{~dB}$.

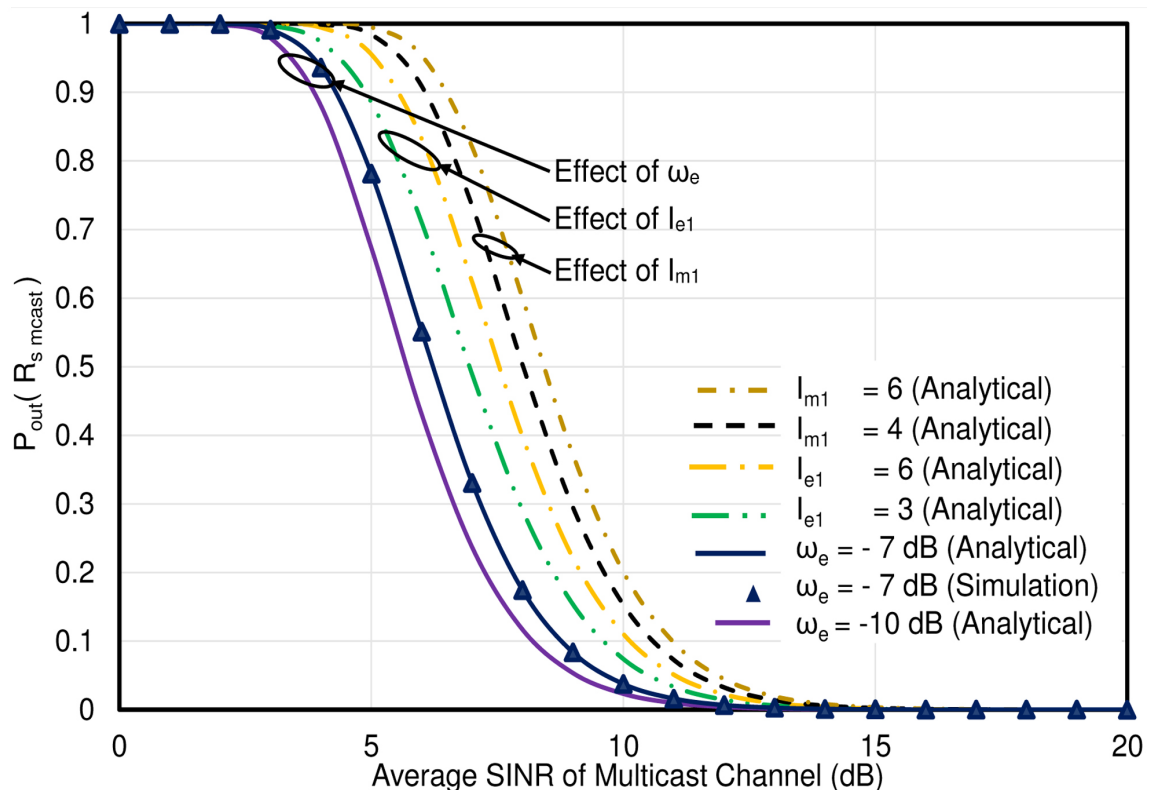

Figure 9. Effect of the SINR of evesdropper channel, $\omega_{e}$, and the effect of interference at the multicast users, $I_{m_{1}}$, and the effect of interference at the eavesdropper, $I_{e_{1}}$, created from Cell-2 on the $P_{\text {out }}\left(R_{\text {smcast }}\right)$ for $I_{m_{1}}=1$ watt and $I_{e_{1}}=3$ watt, $\omega_{e}=-5 \mathrm{~dB}$ and $I_{e_{1}}=3$ watt, and $\omega_{e_{1}}=-5 \mathrm{~dB}$ and $I_{m_{1}}=1$ watt, respectively, with $M=5, N=4$, $\rho_{m_{1}}=0.5, K=2, n_{r_{1}}=n_{e_{1}}=10$ and $m=1.5$. 
concluded that the $P_{\text {out }}\left(R_{\text {smcast }}\right)$ decreases with $I_{e_{1}}$ and increases with $\omega_{e}$ and $I_{m_{1}}$ as one expects.

The $P_{\text {out }}\left(R_{\text {smcast }}\right)$ for Cell-2 is shown in Figure 10 as a function of the average SINR of the multicast channel for selected values of $\omega_{e}, I_{m_{1}}$, and $I_{e_{1}}$. This figure illustrates the effects of $\omega_{e}, I_{m_{1}}$, and $I_{e_{1}}$ on the $P_{\text {out }}\left(R_{\text {smcast }}\right)$ for selected values of system parameters. It is observed that the $P_{\text {out }}\left(R_{\text {smcast }}\right)$ decreases, if the values of $I_{e_{2}}$ increases from 3 watt (indicated by the long dash dot line) to 10 watt (indicated by the long dash dot-dot line) keeping the value of $I_{m_{2}}=2$ watt and $\omega_{e_{2}}=-5 \mathrm{~dB}$, respectively. The $P_{\text {out }}\left(R_{\text {smcast }}\right)$ increases, if the values of $I_{m_{2}}$ increases from 2 watt (indicated by the dash line) to 10 watt (indicated by the solid line) keeping the value of $I_{e_{2}}$ equal to 1 watt. This is because, interference at the eavesdropper of Cell-2 increases the secrecy multicast capacity and interference at the multicast users decreases the secrecy multicast capacity, and the increase in secrecy multicast capacity causes a reduction in the secure outage probability and decrease in secrecy multicast capacity causes an improvement in the secure outage probability. Hence, it can be concluded that the $P_{\text {out }}\left(R_{\text {smcast }}\right)$ decreases with $I_{e_{2}}$ and increases $I_{m_{2}}$.

The $P_{\text {out }}\left(R_{\text {smcast }}\right)$ for Cell-2 is shown in Figure 11 as a function of the average SINR of the multicast channel for selected values of $W, I_{m_{2}}$ and $L$. This figure shows that $P_{\text {out }}\left(R_{\text {smcast }}\right)$ increases with the number of multicast users, $L$, and interference at the multicast users, $I_{m_{2}}$, which means, the secrecy multicast capacity decreases with $L$ and $I_{m_{2}}$. But this reduction in secrecy multicast capacity can be minimized by increasing the number of relays which provides additional diversity to the multicast users.

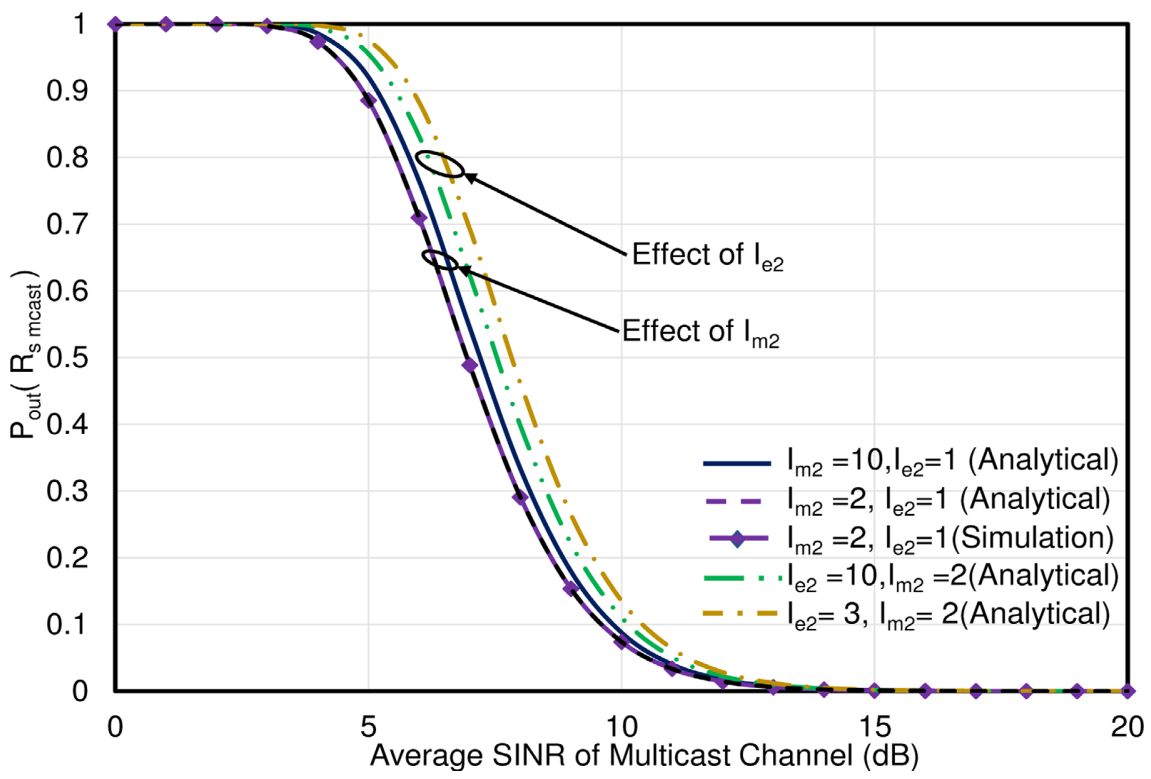

Figure 10. Effect of the interference powers of multicast users of Cell-2, $I_{m_{2}}$, and the interference powers of eavesdropper of Cell-2, $I_{c_{2}}$, generated from Cell-1 on the $P_{\text {out }}\left(R_{\text {smcast }}\right)$ with $I_{e_{2}}=1$ watt, $\omega_{e_{2}}=-5 \mathrm{~dB}, W=5, Q=4, \rho_{m_{2}}=0.5, n_{r_{2}}=n_{e_{2}}=10$ and $m=1.5$. 


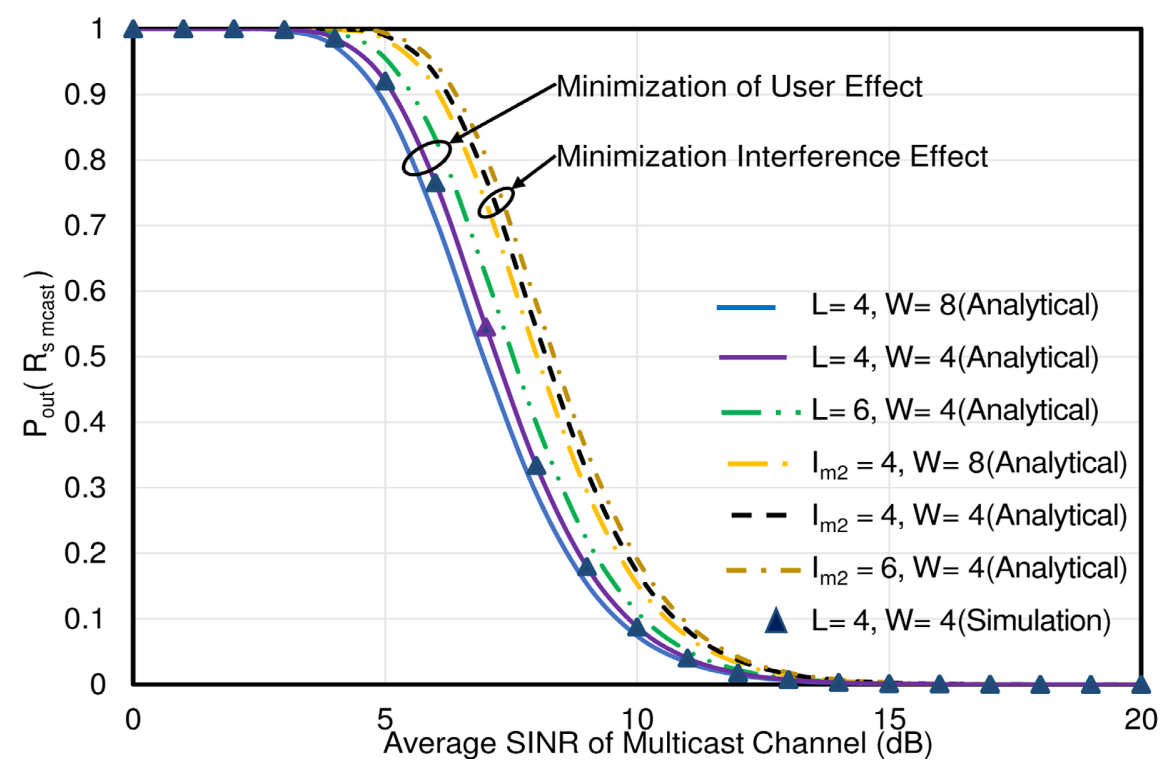

Figure 11. Effect of the number of relays of Cell-2, W, on the $P_{\text {out }}\left(R_{\text {smcast }}\right)$ for selected values of $I_{m_{2}}$ and $L$ with $I_{e_{2}}=1$ watt, $\omega_{e_{2}}=-5 \mathrm{~dB}, Q=4, \rho_{m_{2}}=0.5, n_{r_{2}}=n_{e_{2}}=10$ and $m=1.5$.

Therefore, based on the closed-form analytical expressions for the probability of non-zero secrecy multicast capacity and the secure outage probability for multicasting, and from the observations of numerical results, the main findings of this paper can be summarized as follows: the security in multicasting degrades with the intensity of fading, the number of multicast users, eavesdroppers and their antennas, and due to the antenna correlation and interferences at the multicast users. This problem can be solved by using opportunistic relaying technique in which $K$ relays compete to transmit their received signals with high signal-to-noise ratio (SNR) but only the best relay with the highest SNR is allowed to transmit its received signal to the receivers. Although, the zero-forcing precoding technique is used in the existing literature to mitigate the effect of interference by canceling out the interference power, but the mathematical modeling of this technique is more complex in the case of multi-user multiple-input multiple-out (MIMO) channel and there is no chance to use the interference power to enhance the security in multicasting. The opportunistic relaying is more advantageous than the zero-forcing precoding technique in the sense that, the mathematical modeling of opportunistic relaying is less complex than zero-forcing precoding technique and it reduces the effects of interferences providing additional cooperative diversity gain without canceling out the interference power. Hence, there is a chance to use this interference power to enhance the security in multicasting applying selective and phase-alignment precoding techniques in the best relay.

\section{Conclusion}

This paper focuses on the development of an analytical mathematical model to 
ensure the security in wireless multicasting through multicellular network considering the simultaneous effect of interference and correlation. Based on the mathematical model and the observations of numerical results, it can be concluded that the degradation of security in multicasting occurs due to the severity of fading, the effects of correlation and interference and increasing in the number of multicast users, eavesdroppers and their antennas. This problem of ensuring perfect secrecy in multicasting can be successfully mitigated by using opportunistic relaying technique which is mathematically less complex than other methods used for interference mitigation and easy to understand. Moreover, it paves the way of using interference power to improve the security in multicasting by applying selective and phase-alignment precoding techniques in the best relay.

\section{Conflicts of Interest}

The authors declare no conflicts of interest regarding the publication of this paper.

\section{References}

[1] Chuah, C., Tse, D., Kahn, J. and Valenzuela, R. (2012) Capacity Scaling in MIMO Wireless Systems under Correlated Fading. IEEE Communications Letters, 16, $1600-1603$.

[2] Haenggi, M. (2002) Diversity Loss Due to Interference Correlation. IEEE Transactions on Information Theory, 48, 637-650.

[3] Sarker, D.K., Sarkar, M.Z.I. and Anower, M.S. (2017) Secure Wireless Multicasting with Linear Equalization. Physical Communication, 25, 201-213. https://doi.org/10.1016/j.phycom.2017.07.007

[4] Shrestha, A.P., Jung, J. and Kwak, K.S. (2013) Secure Wireless Multicasting in Presence of Multiple Eavesdroppers. International Symposium on Communications and Information Technologies, Surat Thani, 4-6 September 2013, 814-817. https://doi.org/10.1109/ISCIT.2013.6645929

[5] Giti, J.E., Chowdhury, S.A.H. and Ali, M.M. (2015) Enhancing Security in Wireless Multicasting with Selective Precoding. International Journal of Systems, Control and Communications, 6, 277-291. https://doi.org/10.1504/IJSCC.2015.072515

[6] Giti, J.E., Chowdhury, S.A.H., Rashid, M.M. and Ali, M.M. (2015) Enhancing Secure Wireless Multicasting Using Phase Alignment Relay Precoding. IEEE International Conference on Electrical and Electronics Engineering, Rajshahi, 4-6 November 2015, 189-192. https://doi.org/10.1109/CEEE.2015.7428252

[7] Sayed, R., Sarkar, M.Z.I., Ali, S.M.R. and Sarker, D.K. (2016) Secure Wireless Multicasting through Multi-Cellular MIMO Networks with Linear Equalization. International Conference on Electrical Engineering and Information Communication Technology, Dhaka, 22-24 September 2016, 1-6. https://doi.org/10.1109/CEEICT.2016.7873159

[8] Nguyen, V.-D., Duong, T.Q., Shin, O.-S., Nallanathan, A. and Karagiannidis, G.K. (2017) Enhancing PHY Security of Cooperative Cognitive Radio Multicast Communications. IEEE Transaction on Cognitive Communications and Networking, 3 599-613. https://doi.org/10.1109/TCCN.2017.2748132 
[9] Nguyen, M.-N., Nguyen, N.-P., Costa, D.B.D., Nguyen, H.-K. and Sousa, R.T.D. (2017) Secure Cooperative Half-Duplex Cognitive Radio Networks with $k$-th Best Relay Selection. IEEE Access, 5, 6678-6687. https://doi.org/10.1109/ACCESS.2017.2686984

[10] Kundu, M.K., Badrudduza, A.S.M., Sarkar, M.Z.I. and Sarker, D.K. (2019) Secure Wireless Multicasting with Mixture Gamma Distribution for Generalized-k Fading Channels. IEEE International Conference on Computer, Communication, Chemical, Materials and Electronic Engineering, Rajshahi, 11-12 July 2019, 1-4. https://doi.org/10.1109/IC4ME247184.2019.9036571

[11] Borshon, Z.I., Nafis, A.S.M., Badruduzza, A.S.M., Kundu, M. and Sarkar, M. (2019) Enhancing Wireless Security over Weibull Fading Multicast Channel. IEEE International Conference on Computer, Communication, Chemical, Materials and Electronic Engineering, Rajshahi, 11-12 July 2019, 1-4. https://doi.org/10.1109/IC4ME247184.2019.9036690

[12] Badrudduza, A.S.M. and Kundu, M.K. (2019) Enhancing Security in Wireless Multicasting over Kappa Meu Fading Channel. IEEE International Conference on Computer and Information Technology, Dhaka, 18-20 December 2019, 1-5. https://doi.org/10.1109/ICCIT48885.2019.9085488

[13] Atallah, M. and Kaddoum, G. (2019) Secrecy Analysis in Wireless Network with Passive Eavesdroppers by Using Partial Cooperation. IEEE Transactions on Vehicular Technology, 68, 7225-7230. https://doi.org/10.1109/TVT.2019.2913934

[14] Sultana, R., Sarkar, M.Z.I. and Hossain, M.S. (2019) Enhancing Security in Cognitive Radio Multicast Networks Using Interference Power. Journal of Computer and Communication, 7, 66-78. https://doi.org/10.4236/jcc.2019.712007

[15] Kibria, M., Sarkar, M. and Sarker, D. (2019) Enhancing Security in Multicasting with Opportunistic Relaying over Nakagami-m Fading Channels. Journal of Applied Engineering and Science, 3, 80-90.

[16] Sun, G., Liu, M., Han, Z. and Zhao, C. (2020) Multiple Eavesdropper-Based Physical Layer Security in SIMO System with Antenna Correlation. KSII Transactions on Internet and Information Systems, 14, 422-436. https://doi.org/10.3837/tiis.2020.01.023

[17] Badrudduza, A., Sarkar, M. and Kundu, M. (2020) Enhancing Security in Multicasting through Correlated Nakagami- $m$ Fading Channels with Opportunistic Relaying. Physical Communication, 43, Article ID: 101177. https://doi.org/10.1016/j.phycom.2020.101177

[18] Shahriyer, S., Badrudduza, A., Shabab, S. and Kundu, M. (2021) Opportunistic Relaying in Multicasting over Generalized Shadowed Fading Channels: A PHY Security Analysis.

[19] Duang, T.Q., Bao, V.N.Q. and Zepernick, H.-J. (2009) On the Performance of Selection Decode-and-Forward Multiple Relay Networks over Nakagami- $m$ Fading Channels. IEEE Communications Letters, 13, 172-174. https://doi.org/10.1109/LCOMM.2009.081858

[20] Krikidis, I., Thompson, J.S., Mclaughlin, S. and Goertz, N. (2009) Max-Min Relay Selection for Legacy Amplify-and-Forward Systems with Interference. IEEE Transactions on Wireless Communications, 8, 3016-3027.

https://doi.org/10.1109/TWC.2009.080383

[21] Lin, J.C. and Poor, H.V. (2021) Optimum Combiner for Spatially Correlated Nakagami-m Fading Channels. IEEE Transactions on Wireless Communications, 20, 771-784. https://doi.org/10.1109/TWC.2020.3028284 
[22] Kumar, N. and Bhatia, V. (2015) Performance Analysis of Amplify-and-Forward Cooperative Networks with Best-Relay Selection over Weibull Fading Channels. Wireless Personal Communications, 85, 1-13.

https://doi.org/10.1007/s11277-015-2799-y

[23] Gradshteyn, I.S. and Ryzhik, I.M. (2007) Table of Integrals, Series, and Products. 7th Edition, Academic Press, San Diego.

[24] Khisti, A. and Zhang, D. (2013) Artificial-Noise Alignment for Secure Multicast Using Multiple Antennas. IEEE Communications Letters, 17, 1568-1571. https://doi.org/10.1109/LCOMM.2013.060513.130802

[25] Sarkar, M.Z.I. and Ratnarajah, T. (2011) Secure Wireless Multicasting through Nakagami-m Fading MISO Channel. 2011 Conference Record of the 45 th ASILOMAR Conference on Signals, Systems and Computers, Pacific Grove, 6-11 November 2011, 300-304. https://doi.org/10.1109/ACSSC.2011.6190006 